\title{
The galactic plane region near $\ell=93^{\circ}$
}

\section{HII region NRAO 655}

\author{
T. Foster ${ }^{1}$ and D. Routledge ${ }^{2}$ \\ 1 Dept. of Physics, University of Alberta, Edmonton, Canada \\ 2 Dept. of Electrical and Computer Engineering, University of Alberta, Edmonton, Canada
}

Received 29 August 2000 / Accepted 24 November 2000

\begin{abstract}
We present new Canadian Galactic Plane Survey radio continuum and $\lambda 21 \mathrm{~cm} \mathrm{HI}$ line observations of NRAO 655 (G93.4+1.8), plus radio recombination line observations, and optical $\mathrm{H} \alpha$-line observations. The radio spectrum of NRAO 655 confirms its emission as thermal. From the $\lambda 21 \mathrm{~cm}$ HI data we find an atomic hydrogen cavity associated with this object at $v \simeq-71.5 \mathrm{~km} \mathrm{~s}^{-1}$. The cavity corresponds in position and size to the brightest radio continuum emission from NRAO 655. The corresponding kinematic distance is $8.8 \mathrm{kpc}$, placing NRAO 655 in the Perseus Arm. NRAO 655's linear size is therefore $70 \mathrm{pc} \times 130 \mathrm{pc}$. To confirm the $\lambda 21 \mathrm{~cm}$ HI velocity we present the first recombination line detection of NRAO 655 (H158 line, $v \simeq-71 \mathrm{~km} \mathrm{~s}^{-1}$ ), and the first observations of a molecular cloud coinciding with NRAO 655 (at $v \simeq-72 \mathrm{~km} \mathrm{~s}^{-1}$ ). The first optical detection of $\lambda 656 \mathrm{~nm} \mathrm{H \alpha}$ emission line features in NRAO 655 is also presented, and the $\mathrm{H} \alpha$ emission line brightness is determined. We suggest that the eastward extension of this strongly asymmetric object originates in a champagne outflow, and we estimate its age. We show that a single early-type star cannot be responsible for the outflow, whereas a group of later-type stars would suffice. A partial HI shell is seen adjacent to the brightest part of NRAO 655; we suggest that it has been formed by dissociation of $\mathrm{H}_{2}$ in the molecular cloud.
\end{abstract}

Key words. HII regions - radio continuum: ISM - radio lines: ISM - ISM: jets and outflows - ISM: individual objects: NRAO 655

\section{Introduction}

Known Galactic radio sources in the neighbourhood of NRAO 655 (G93.4+1.8) are represented schematically in Fig. 1 and listed in Table 1. Figure 1 is based on $408 \mathrm{MHz}$ observations detailed below. Of the eleven objects labelled, CTB 104A and 3C 434.1 are known to be supernova remnants (SNR's). Their distances are unknown since the only estimates made to date have been based on the $\Sigma-\mathrm{D}$ relation, which is considered by most authors to be inaccurate (Green 1984, 1991). The other nine objects are all thought to be thermal emitters, although NRAO 655 has been classified as an SNR by some authors (see below). Of these nine thermal emitters, however, only BG $2107+49$ (an unusual object which includes a stellar wind shell) and G92.7+3.1 have published radial velocities, obtained from hydrogen recombination line, HI line, and molecular line observations. An unpublished $\mathrm{H} 109 \alpha$ observation by Fich (1983) is also available for the large HII region CTB 102 (G93.1+2.8). Besides these three, therefore, none of the eleven objects in Fig. 1 and Table 1 has a reliable

Send offprint requests to: D. Routledge,

e-mail: routle@ee.ualberta.ca distance estimate. BG $2107+49$ is known to be at a large distance ( $10 \mathrm{kpc}$ kinematic distance from $\mathrm{H} 112 \alpha$ recombination line, ${ }^{12} \mathrm{CO}(1-0)$ line, and $\lambda 21 \mathrm{~cm}$ HI line data; van der Werf \& Higgs 1990), though clearly others such as G92.7+3.1 $\left(v_{\mathrm{LSR}} \simeq-6.5 \mathrm{~km} \mathrm{~s}^{-1}\right)$ and CTB $104 \mathrm{~A}$ (large angular size) are much nearer the Sun. We have chosen to study NRAO 655, and one of the chief goals of this paper is therefore to establish its distance, and hence its physical size. In a later paper (Foster \& Routledge, in prep.) we study the neighbouring SNR 3C 434.1.

Velusamy \& Kundu (1974) mapped NRAO 655 at $2.7 \mathrm{GHz}$ with $5.5^{\prime}$ resolution and at $5 \mathrm{GHz}$ with $6^{\prime}$ resolution. They found a spectral index of $\alpha=-0.3$ (where $S \propto f^{\alpha}$ ) and suggested that NRAO 655 was a possible SNR. On the basis of observations at $1720 \mathrm{MHz}$ with $7.6^{\prime}$ resolution, Mantovani et al. (1982) calculated $\alpha=-0.30 \pm 0.05$ and concluded that NRAO 655 should still be considered as a possible SNR. However, Goss et al. (1984) made observations at $608.5 \mathrm{MHz}$ with $1.7^{\prime}$ resolution and at $4.75 \mathrm{GHz}$ with $2.4^{\prime}$ resolution, and found $\alpha=-0.04 \pm 0.03$. On this basis, and because the source was found to be unpolarized, they concluded that 




GALACTIC LONGITUDE

Fig. 1. Radio sources in the vicinity of NRAO 655. This diagram was produced by contouring the aperture synthesis $408 \mathrm{MHz}$ image of the CGPS J8 field centred on $(l, b)=(93.47,1.00)$. The lowest contour shown is $116 \mathrm{~K}$ of brightness temperature. The sources labelled are also listed in Table 1. Reliable distances are known for only BG 2107+49, G92.7+3.1, and CTB 102

Table 1. HII regions and supernova remnants in Fig. 1. Velocities are published for two objects: $\mathrm{CO}$ and $\mathrm{NH}_{3}$ velocities for WB43, and $\mathrm{H} 112 \alpha,{ }^{12} \mathrm{CO}(1-0)$, and HI velocities for BG $2107+49$. The H109 $\alpha$ velocity for CTB 102 is unpublished

\begin{tabular}{ccccc}
\hline Name & Alias & Type & $v_{\text {LSR }}\left(\mathrm{km} \mathrm{s}^{-1}\right)$ & References \\
\hline G93.1+2.8 & CTB 102 & HII & $v=-58$ & $1,2,3$ \\
G92.7+3.1 & WB43, Mol 133 & HII & $v=-6.1,-6.5,-6.8$ & $4,5,6$ \\
G94.4+2.6 & KR6 & & & 7,8 \\
G93.7+2.6 & KR4 & HII & & $7,8,9$ \\
G93.0+2.0 & NRAO 652 & & & 10,11 \\
G92.3+1.5 & & HII & & 12 \\
G93.9+1.6 & Mol 135, WB93 & SNR & & 4 \\
G94.3-0.3 & CTB 104A & HII & & 11 \\
G93.4+1.8 & NRAO 655 & SNR & & 11,13 \\
G94.0+1.0 & 3C 434.1 & HII complex & $v=-80 \pm 1$ & 12 \\
G91.11+1.58 & BG 2107+49 & &
\end{tabular}

References: (1) Galt \& Kennedy 1968; (2) van der Werf \& Higgs 1991; (3) Fich 1983; (4) Molinari et al. 1996; (5) Miralles et al. 1994; (6) Wouterloot \& Brand 1989; (7) Fich 1986; (8) Kallas \& Reich 1980; (9) Reich et al. 1990a; (10) Pauliny-Toth et al. 1966; (11) Mantovani et al. 1982; (12) van der Werf \& Higgs 1990; (13) Landecker et al. 1985.

NRAO 655 was most likely an HII region, with a small possibility remaining that it might be an SNR with an unusually flat spectrum. They suggested that recombination-line observations would provide a definitive test to decide the issue. Goss et al. also commented that if NRAO 655 were indeed an HII region, its elongated structure eastwards might be the result of a champagne outflow (see below). Arendt (1989) continued to classify NRAO 655 with other Galactic SNR's, however, but noted the impossibility of distinguishing SNR's from other Galactic sources solely 
Table 2. Parameters of observations made with DRAO Synthesis Telescope as part of Canadian Galactic Plane Survey

\begin{tabular}{|c|c|c|}
\hline SYNTHESIS & TELESCOPE & OBSERVATIONS \\
\hline parameter & & value \\
\hline 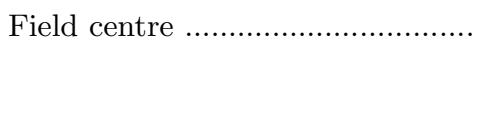 & 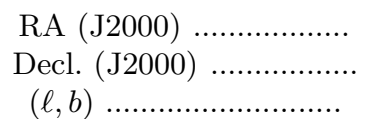 & $\begin{array}{c}21 \mathrm{~h} 22 \mathrm{~m} 36.7 \mathrm{~s} \\
51^{\circ} 30^{\prime} 33^{\prime \prime} \\
93.4662^{\circ}, 1.0000^{\circ}\end{array}$ \\
\hline Date of observations & (2) & 1998 April \\
\hline  & $\begin{array}{l}408 \mathrm{MHz} \ldots \ldots \ldots \ldots \ldots \ldots \ldots \\
1420 \mathrm{MHz} \ldots \ldots \ldots \ldots \ldots \ldots \ldots\end{array}$ & $\begin{array}{l}8.1^{\circ} \text { to } 20 \% \\
2.6^{\circ} \text { to } 20 \%\end{array}$ \\
\hline Synthesised beams $(\mathrm{EW} \times \mathrm{NS}) \ldots$ & $\begin{array}{l}408 \mathrm{MHz} . \ldots \ldots \ldots \ldots \ldots \ldots . . . \\
1420 \mathrm{MHz} \text { continuum ... } \\
\lambda 21 \mathrm{~cm} \text { HI line ............. }\end{array}$ & $\begin{array}{l}3.4^{\prime} \times 4.2^{\prime} \\
58^{\prime \prime} \times 73^{\prime \prime} \\
60^{\prime \prime} \times 76^{\prime \prime}\end{array}$ \\
\hline Baseline coverage &  & $\begin{array}{r}17 \lambda-822 \lambda \\
61 \lambda-2864 \lambda\end{array}$ \\
\hline Baseline increment ...................... & (2) & $4.286 \mathrm{~m}$ \\
\hline 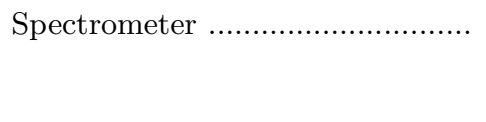 & 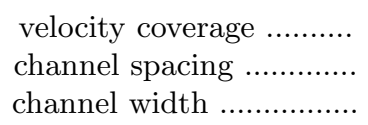 & $\begin{array}{c}-165-45 \mathrm{~km} \mathrm{~s}^{-1} \\
0.824 \mathrm{~km} \mathrm{~s}^{-1} \\
1.32 \mathrm{~km} \mathrm{~s}^{-1}\end{array}$ \\
\hline RMS noise, $T_{b}$, map centre....... & $\begin{array}{l}408 \mathrm{MHz} \ldots \ldots \ldots \ldots \ldots \ldots \ldots . . . \\
1420 \mathrm{MHz} . \ldots \ldots \ldots \ldots \ldots \ldots \ldots \\
\text { 1-channel HI map .......... }\end{array}$ & $\begin{array}{c}524 \mathrm{mK} \\
38 \mathrm{mK} \\
2.7 \mathrm{~K}\end{array}$ \\
\hline Polarisation ....... & $\begin{array}{l}408 \mathrm{MHz} \ldots \ldots \ldots \ldots \ldots \ldots \ldots \\
1420 \mathrm{MHz} \ldots \ldots \ldots \ldots \ldots \ldots \ldots \\
\lambda 21 \mathrm{~cm} \text { HI line } \ldots \ldots \ldots \ldots \ldots . . . . . .\end{array}$ & $\begin{array}{c}\text { RHCP } \\
\text { Stokes } I, Q, U \\
\text { RHCP and LHCP }\end{array}$ \\
\hline
\end{tabular}

on the basis of their infrared colours. No optical detection or radio recombination line detection of NRAO 655 has been reported to date. We report the first of both of these in this paper, as well as $\mathrm{HI}$ and $\mathrm{CO}$ investigations.

\section{Observations and data reduction}

\subsection{Aperture synthesis radio imaging}

The field of NRAO 655 was observed at $1420 \mathrm{MHz}$ and $408 \mathrm{MHz}$ with the Synthesis Telescope at the Dominion Radio Astrophysical Observatory (DRAO). These continuum and $\lambda 21 \mathrm{~cm}$ atomic hydrogen line observations were carried out as part of the J8 field of the Canadian Galactic Plane Survey (CGPS). The CGPS is a project by a consortium of researchers from five countries to map a large segment of the northern Galactic plane in radio and infrared wavelengths at a resolution approaching $1^{\prime}$ (Taylor et al. $2000)$. The J8 field centre is $(\ell, b)=(93.4662,1.0000)$. The DRAO Synthesis Telescope is an array of seven $9 \mathrm{~m}$ diameter telescopes, positioned along a $600 \mathrm{~m}$ EW baseline. It produces wide-field images of $1^{\prime}$ and $3.4^{\prime}$ resolution at $1420 \mathrm{MHz}$ and $408 \mathrm{MHz}$, respectively. The data for these images are combined, after addition of low-order spatial frequency data from single-dish telescopes, to form the CGPS mosaics. The HI images are taken in 256 channels separated by $0.824 \mathrm{~km} \mathrm{~s}^{-1}$. Other relevant parameters of the telescope are given in Table 2, and more detail is available in Landecker et al. (2000). Several 12-hour observations at uniform antenna spacings are taken to produce a fully sampled $u v$ plane at both continuum frequencies and in the $\lambda 21 \mathrm{~cm} \mathrm{HI}$ line out to the baseline limit, except for antenna spacings shorter than $12.9 \mathrm{~m}$. The primary calibrators for the $1420 \mathrm{MHz}$ and $408 \mathrm{MHz}$ continuum observations were 3C 295, 3C 286, and 3C 147; flux densities for these sources were taken from Baars et al. (1977).

This paper deals with data from the CGPS J8 field alone, with no contributions from adjacent fields to the data. Polar-diagram correction was applied to level the gain across the J8 field, which varied due to the beamshape of the individual antennas. This was applied to both continuum and HI line data, and all images in this paper have been corrected in this way. Continuum images were CLEANed, using the algorithm of Clark (1980) for smalldiameter sources and Steer et al. (1984) for extended structure. Self-calibration of the continuum maps was performed. Because of the almost complete coverage of the $u v$-plane, the sidelobe levels of the uncleaned beam are low (first sidelobe $=-3.6 \%$; second sidelobe $=+2.2 \%$ ), and it was not necessary to CLEAN the HI images.

Large, diffuse structures present in the field of view are not visible to the Synthesis Telescope, since antenna spacings shorter than $12.9 \mathrm{~m}$ are not obtained. The missing short-spacing information was obtained for $1420 \mathrm{MHz}$ from the Effelsberg $21 \mathrm{~cm}$ Galactic Plane Survey (Reich et al. 1990b), for $408 \mathrm{MHz}$ from the $408 \mathrm{MHz}$ All-Sky Survey (Haslam et al. 1982), and for HI spectral line images from an all-sky HI survey made with the observatory's 25.6 m paraboloid (Higgs 1999; Higgs \& Tapping 2000). Images from these single-dish surveys and the 

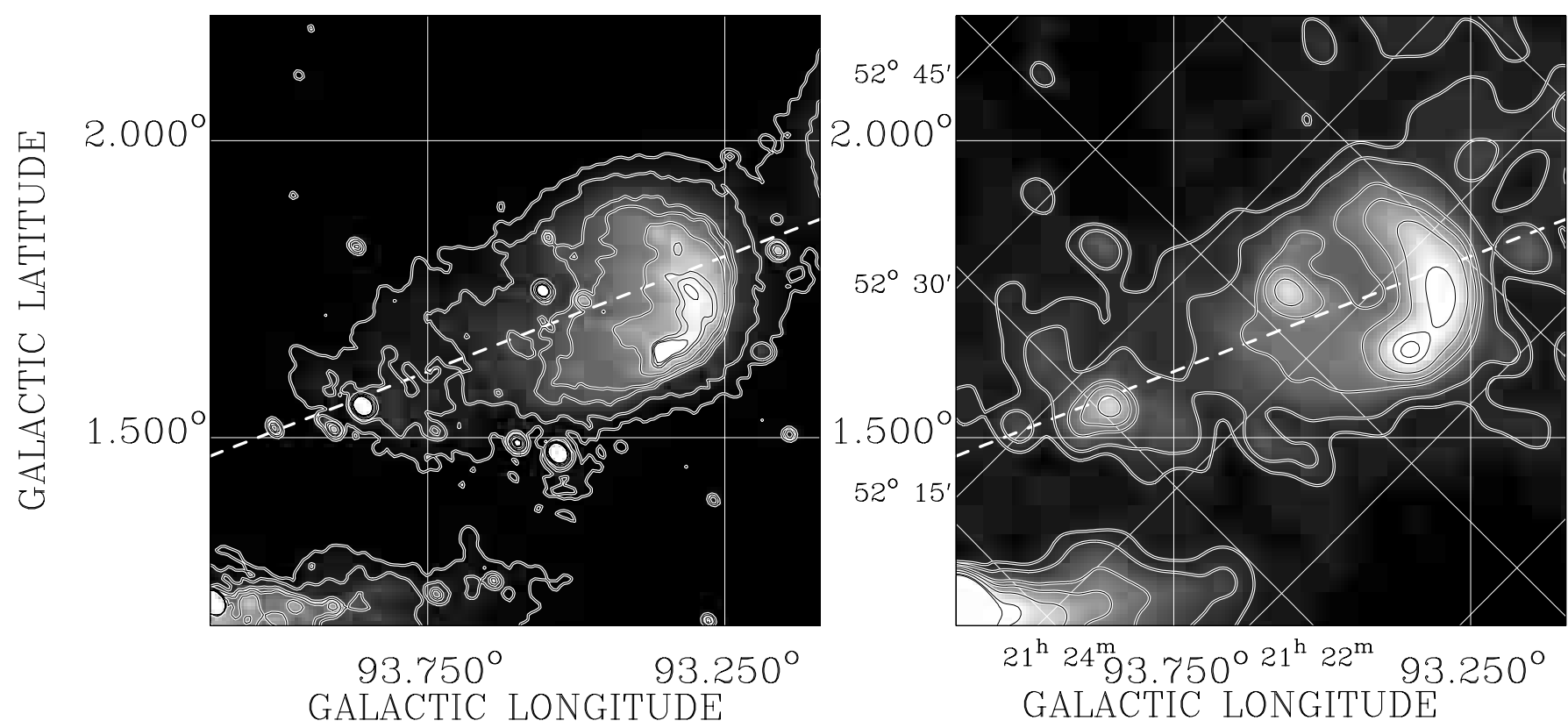

Fig. 2. The $1420 \mathrm{MHz}$ (left) and $408 \mathrm{MHz}$ (right) continuum brightness temperature of NRAO 655, with the small-diameter source G93.9+1.6 within the boundaries of NRAO 655 in the East. Contour levels at $1420 \mathrm{MHz}$ are 1.5, 2, 3, 3.5, 4.5, 6, 6.5, and $7 \mathrm{~K}$ and those at $408 \mathrm{MHz}$ are 113.5, 120, 135, 145, 160, 180, and $190 \mathrm{~K}$. The $(\alpha, \delta)$ grid in the $408 \mathrm{MHz}$ image is in J2000 coordinates. The dashed white line in each image indicates the approximate axis of symmetry of NRAO 655 . In each image the circular head, the tail, and the bright kernel are seen

Synthesis Telescope images were added together with complementary $u v$-plane tapering so that a smooth transition between data sets was achieved in the $u v$-plane. That is, at a low cut-off spatial frequency the single-dish data were given full weight while the Synthesis Telescope data were given zero weight, and vice versa at a high cut-off spatial frequency. Linear weighting functions were applied to the two data sets between these two cut-off radii in the $u v$ plane. This process ensures that the radio continuum and HI-line images in this paper portray all structures accurately, including those of large angular scale.

Continuum emission has been removed from the HI images in this paper. This was accomplished by the CGPS processing team by forming a $1420 \mathrm{MHz}$ continuum image from those end channels of the 256-channel spectrometer which were "empty" of line emission, and subtracting this image from each spectral channel.

\subsection{H158 $\alpha$ recombination-line observations}

Observations of $\mathrm{H} 158 \alpha$ emission at $1651.541 \mathrm{MHz}$ were undertaken using the $25.6 \mathrm{~m}$ paraboloid at DRAO. The beamwidth of the $25.6 \mathrm{~m}$ telescope at this frequency is $30^{\prime}$ $F W H M$, and its aperture efficiency $\simeq 53$ percent. $T_{\text {system }} \simeq$ $50 \mathrm{~K}$ excluding sky contributions. Two orthogonal linear polarizations were received. The channel spacing in the 256-channel digital autocorrelation spectrometer was $1.418 \mathrm{~km} \mathrm{~s}^{-1}$, and the channel width was $2.27 \mathrm{~km} \mathrm{~s}^{-1}$. The observation was centred on $(\ell, b)=(93.37,1.75)$, which is the brightest portion of NRAO 655 (see Fig. 2). Frequency switching by $2 \mathrm{MHz}$ was employed. Since the useable receiver bandwidth was $4 \mathrm{MHz}$, this resulted in no loss of observing time, and the two frequency-offset spectra were subsequently shifted and subtracted to produce the final output spectrum. Very narrow-band interference, possibly of satellite origin, appeared in the form of spikes in the output spectrum which were of sufficiently low level that they could be identified and removed only in the final spectrum. A positive $(6.4 \sigma)$ detection of H158 $\alpha$ emission from NRAO655 was achieved with a cumulative integration time of 40 hours (see below).

\subsection{Optical observations}

No optical detection of any object in Table 1, other than BG 2107+49 (Higgs et al. 1987) has been reported in the literature. We present here the first detection of NRAO 655 in $\mathrm{H} \alpha+[\mathrm{NII}]$ emission (see Fig. 10). The observations were made with the University of Alberta's $0.5 \mathrm{~m}$ telescope, which has a CCD camera equipped with a cooled TK512 chip $(512 \times 512$ pixels $)$ operating at the prime focus. Auxiliary optics produce an effective $f /-$ ratio of 3.45 , giving a field of view of $27^{\prime} \times 27^{\prime}$. The precision equatorial drive permits long guided exposures with the field of view at fixed position angle. The interference filter used for this observation has central wavelength $656 \mathrm{~nm}$ and width $7.5 \mathrm{~nm}$, passing the $\lambda 656 \mathrm{~nm}$ $\mathrm{H} \alpha$ line and also the [NII] lines at $\lambda 655 \mathrm{~nm}$ and $\lambda 658 \mathrm{~nm}$. Cooled to $263 \mathrm{~K}$, the CCD camera has 90 percent quantum efficiency at $550 \mathrm{~nm}$ wavelength, gain of 4.6 electrons per analog-digital unit (adu), measured read-noise 
of 30 electrons, and thermal generation rate of 1.2 electrons $\mathrm{s}^{-1}$ pixel $^{-1}$ when multi-pinned phasing (MPP) is implemented. The response of the CCD camera was found to be linear to better than $0.5 \%$ over the range $100 \mathrm{adu}$ to $62000 \mathrm{adu}$. The field centre for the $\mathrm{H} \alpha+[\mathrm{NII}]$ observations was $(\alpha, \delta)=\left(21 \mathrm{~h} 18 \mathrm{~m} 15 \mathrm{~s}, 55^{\circ} 55^{\prime} 40^{\prime \prime}\right)$ in $\mathrm{J} 2000$ coordinates. Flat fields were obtained through the filter near the zenith just after sunset to 80 percent of the full well level of $65535 \mathrm{adu}$; at least 15 flat fields were taken, separated by small position shifts to relocate stars in the frame. At least six "dark" images of the CCD chip's thermally generated signal were also made, of duration equal to that of the actual target frames which followed. These were $600 \mathrm{~s}$ each. The 22 target frames on NRAO 655 were scaled to a common airmass, and star centres aligned. A composite image was made, the exposure time of which totalled $13200 \mathrm{~s}$. Individual observations spanned a range of airmasses from 1.0 to 1.4. An extinction coefficient was derived from observations of planetary nebulae, and was used to correct the composite image to zero airmass.

To calibrate the $\mathrm{H} \alpha+[\mathrm{NII}]$ flux, observations of the planetary nebula NGC 6720 were taken through the same interference filter to derive a gain calibration factor. The calculation used the zero-airmass intercept and the published $\mathrm{H} \alpha+[\mathrm{NII}]$ brightness of NGC 6720 (Hawley \& Miller 1977). This brightness was $5.410^{-6} \mathrm{~W} \mathrm{~m}^{-2} \mathrm{sr}^{-1}$. A large number of faint stars were removed by using the IRAF procedure DAOPHOT (Stetson 1987) before the image was convolved to $1^{\prime}$ resolution to improve the signalto-noise level of extended nebulous emission. The resulting rms noise level in areas without such nebulosity was found to be $\sigma=1.1510^{-18} \mathrm{~W} \mathrm{~m}^{-2}$.

\section{Results}

Figure 2 shows the 1420 and $408 \mathrm{MHz}$ continuum images of NRAO 655. The angular resolution of the two images is $1^{\prime} \times 1.2^{\prime}$ and $3.4^{\prime} \times 4.2^{\prime}$, respectively. In each, NRAO 655 appears to have an axis of approximate symmetry which lies roughly parallel (within $\sim 20^{\circ}$ ) to the Galactic plane, but on the other hand the object is very asymmetrical along this axis. We use the following terminology and dimensions, based on the $1420 \mathrm{MHz}$ image: there is a "head" to the West with an approximately circular outline ( diameter $\simeq 26^{\prime}$ ), a "tail" to the East of length $\simeq 38^{\prime}$ from the centre of the head, and within the head there is a brighter roughly semi-circular area (diameter $\simeq 19^{\prime}$ ) which we shall refer to as the "kernel". Figure 3 shows a $1420 \mathrm{MHz}$ brightness-temperature profile taken along the symmetry axis of NRAO 655, shown by the white dashed line in Fig. 2. The brightness in the tail drops off slowly toward the East (disregarding point sources). The brightest part of the kernel is in the West, and the brightness of the kernel decreases extremely rapidly on the west side. We show below that it is on the west side that the ionized gas in the kernel rapidly gives way to atomic gas, and then to molecular gas in an adjacent molecular cloud.

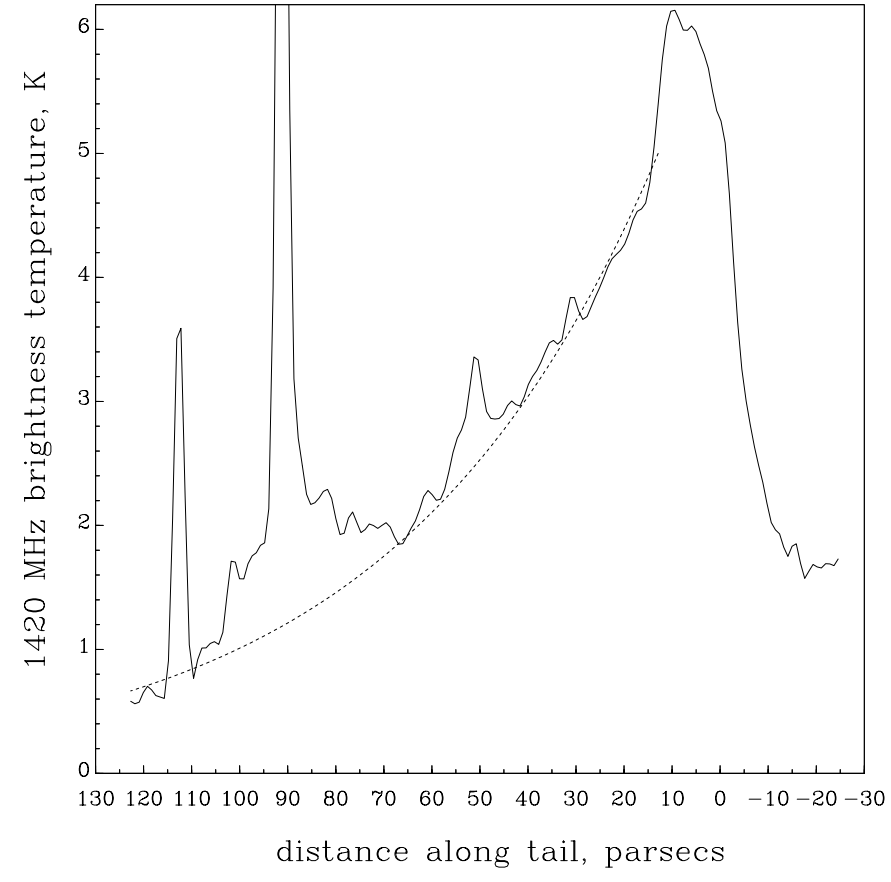

Fig. 3. Brightness temperature profile at $1420 \mathrm{MHz}$ (solid line) taken along the dashed white lines shown in Fig. 2. Placement of the origin of the distance scale is somewhat arbitrary. The brightness of the kernel decreases gradually in the East and very rapidly in the West, and the two sharp peaks at the left are caused by small-diameter sources. The dashed line shows the function $5 \mathrm{e}^{-0.018(r-12)}$ which was fitted by eye to the observed $T_{b}$ profile

The infrared appearance of NRAO 655 is shown in Fig. 4, which is the $60-\mu \mathrm{m}$ HIRES image (Cao et al. 1997). Contours of radio continuum emission have been superimposed which show that the infrared morphology is very similar to that of the radio continuum. The "kernel" is clearly seen in the infrared image, as is the lowerbrightness tail to the East, though the outline of the head is confused with other infrared emission and localised regions of high infrared intensity are seen in the tail. IRAS $21202+5157$ coincides with the intense radio source G93.9+1.6 (see Fig. 2). This may be a foregound or background source, or could possibly be related to a collision of the NRAO 655 champagne outflow (see below) with density inhomogeneities in the intercloud medium (Tenorio-Tagle \& Bedijn 1981). Such speculations call for high-resolution recombination-line observations of the area, and will not be pursued further here. There is a suggestion of an arc of infrared brightness on the east side of the roughly circular head (see also Fig. 10b), but this arc is at least partly comprised of image processing artifacts centred on small-diameter sources. The radio source at $(\ell, b)=(93.53,1.47)$ is IRAS $21190+5140$.

The $\mathrm{H} 158 \alpha$ recombination line observations of the head of NRAO 655 produced the spectrum shown in Fig. 5. A Gaussian of $F W H M=40 \mathrm{~km} \mathrm{~s}^{-1}$ has been fitted to the data after removal of a cubic baseline; the amplitude of the Gaussian is 6.4 times the rms noise level 




Fig. 4. The HIRES $60 \mu \mathrm{m}$ image of the NRAO 655 field. The greyscale runs from 40 (dark) to 80 (light) $\mathrm{MJy} \mathrm{sr}^{-1}$ on a linear scale. The contours show 1.5, 3, and $5 \mathrm{~K}$ of $1420 \mathrm{MHz}$ continuuum brightness temperature for comparison. The infrared morphology is seen to match the radio morphology of head, tail, and bright kernel quite closely. Some processing artifacts (crosses) are visible near small-diameter infrared sources. The $(\alpha, \delta)$ grid is in J2000 coordinates

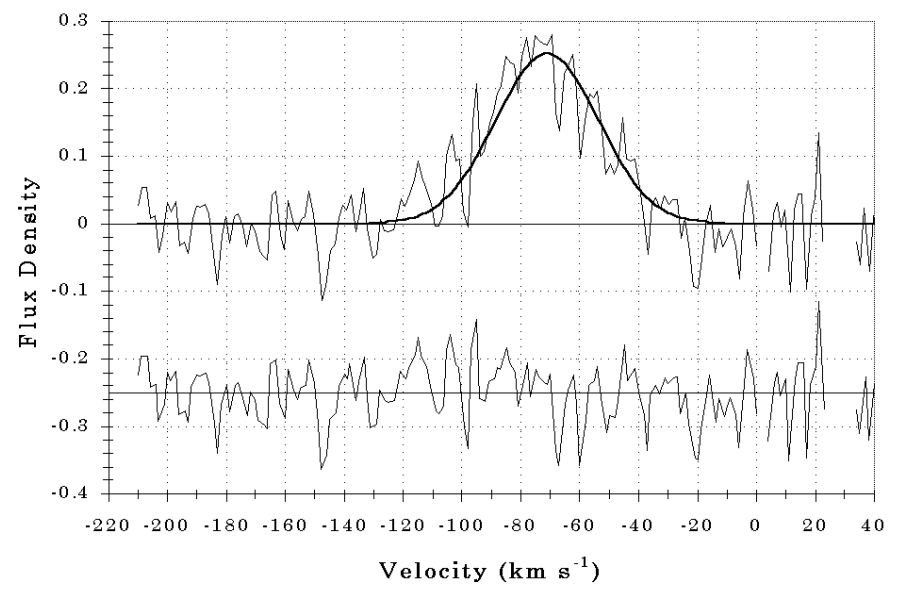

Fig. 5. The $H 158 \alpha$ recombination line spectrum (top) of the head of NRAO 655 after subtraction of a cubic baseline. The Gaussian fitted by least-squares between -110 and $-30 \mathrm{~km} \mathrm{~s}^{-1}$ is shown (top) and the residuals after removal of this Gaussian are also shown (bottom). Narrow interference spikes have been excised at two positive velocities. This is the first recombination-line detection of NRAO 655

in the wings, and its central velocity is $-71 \mathrm{~km} \mathrm{~s}^{-1}$. (All velocities in this paper are relative to the local standard of rest.) The residuals produced by subtracting the Gaussian from the baseline-removed data are also shown. Because of the large width of the line and the corresponding uncertainty in the baseline, the central velocity and amplitude must be taken as only approximate values. However, this is the first recombination-line detection of NRAO 655 and it confirms the thermal nature of this object, though it was originally classified as a supernova remnant (see above). The brightness temperature of the $\mathrm{H} 158 \alpha$ line in this observation is found to be $23 \mathrm{mK}$ and the line-to-continuum ratio is 0.021 .

Figure 6 is a montage of eight velocity channels of ${ }^{12} \mathrm{CO}(1-0)$ emission (Dame et al. 2000), with the $1420 \mathrm{MHz}$ continuum emission from NRAO 655 displayed as contours. (No CO data is available above $b=2^{\circ}$, which is the extent of the survey.) The brightest ${ }^{12} \mathrm{CO}$ emission ( $0.81 \mathrm{~K}$ peak brightness temperature near $-72 \mathrm{~km} \mathrm{~s}^{-1}$ ) coincides with the steep western edge of the continuum emission from the kernel. The $\mathrm{CO}$ emission with this angular resolution $\left(8.7^{\prime} F W H M\right)$ appears to consist of discrete concentrations of brightness, and the position of the peak CO emission changes from channel to channel. As velocity becomes less negative, the position of peak CO brightness moves generally clockwise around the periphery of the head of NRAO 655. As a result, the velocityintegrated $\mathrm{CO}$ emission forms a rough crescent around the 
T. Foster and D. Routledge: The galactic plane region near $\ell=93^{\circ}$. I.


Fig. 6. Eight velocity channels of ${ }^{12} \mathrm{CO}(1-0)$ emission (Dame et al. 2000) spaced at $1.3 \mathrm{~km} \mathrm{~s}^{-1}$ intervals, with greyscale running from 0 (dark) to 0.65 (light) $\mathrm{K}$ of brightness temperature on a linear scale. Bright $\mathrm{CO}$ emission in this velocity range appears in a crescent following the west side of the head of NRAO 655 


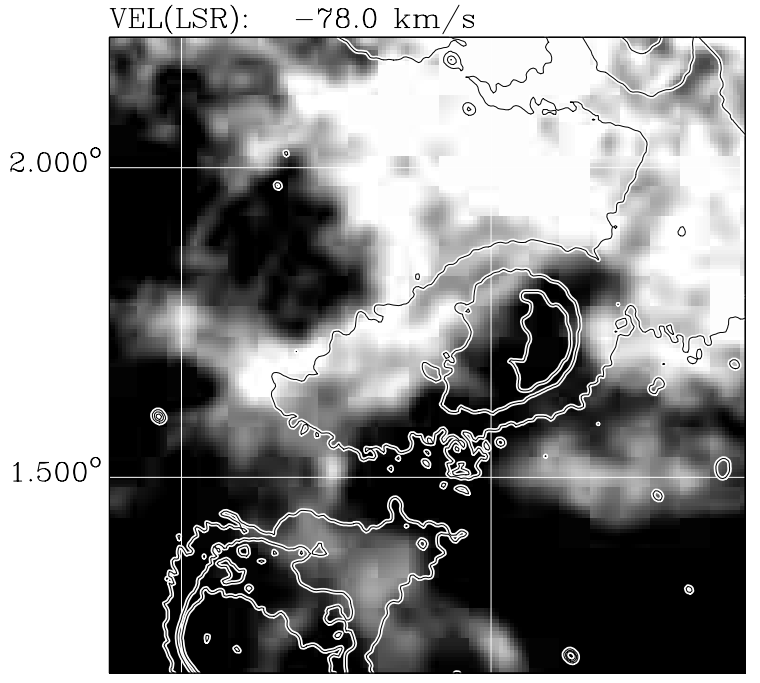

$94.000^{\circ}$

$93.500^{\circ}$

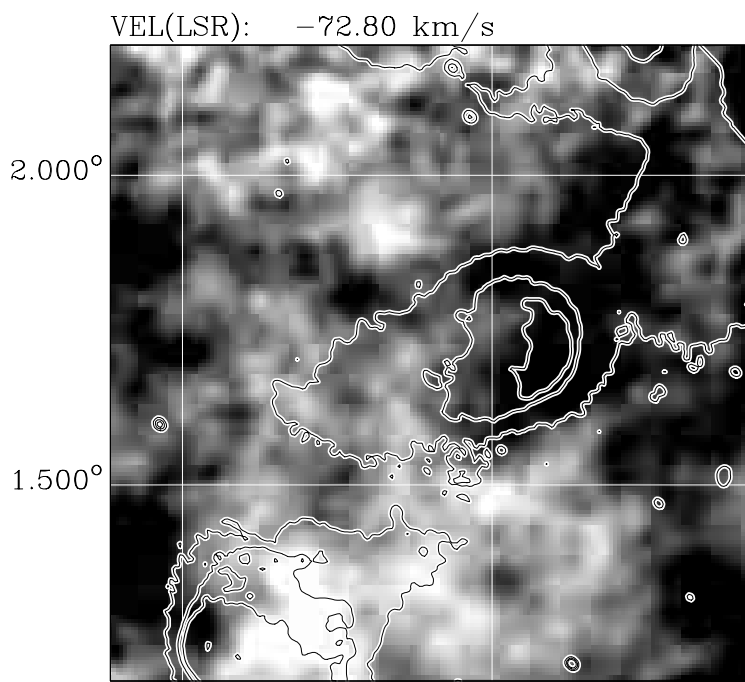

$94.000^{\circ}$

$93.500^{\circ}$

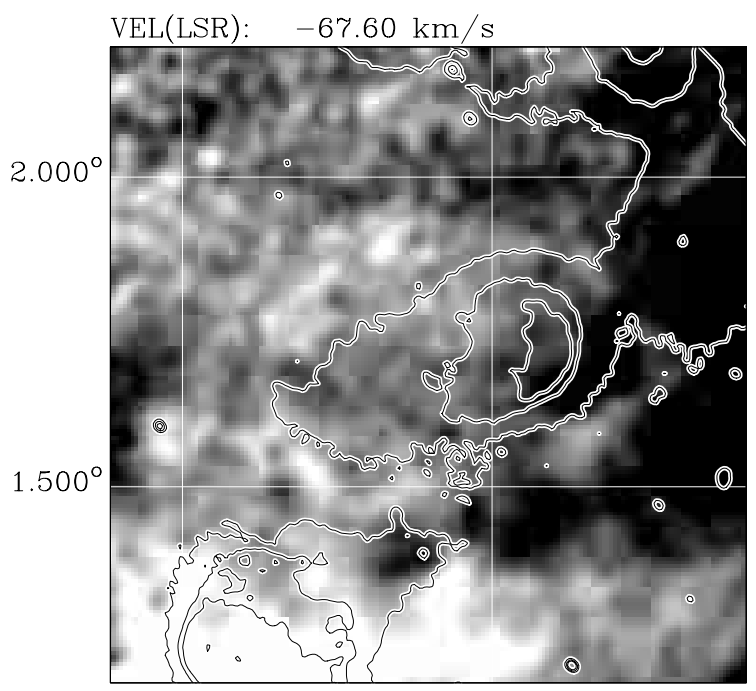

$94.000^{\circ}$

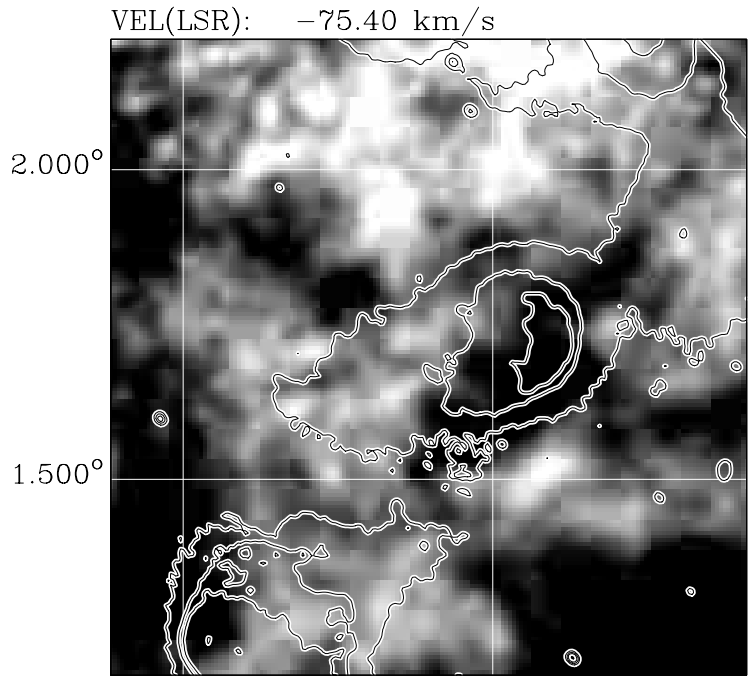

$94.000^{\circ}$

$93.500^{\circ}$

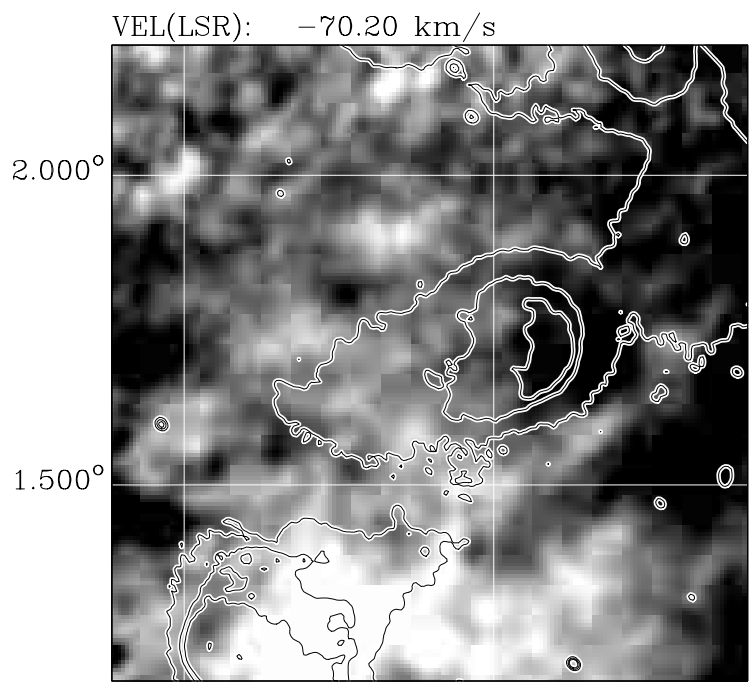

$94.000^{\circ}$

$93.500^{\circ}$

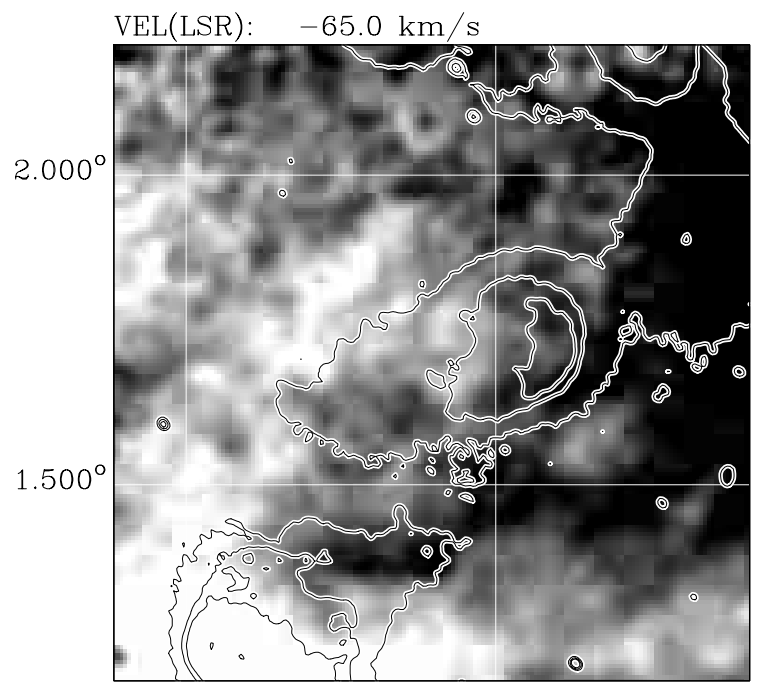

$94.000^{\circ}$

$93.500^{\circ}$

Fig. 7. Six velocity channels of $\lambda 21 \mathrm{~cm} \mathrm{HI} \mathrm{emission} \mathrm{spaced} \mathrm{at} 2.6 \mathrm{~km} \mathrm{~s}^{-1}$ intervals, with greyscale running from -15 (dark) to 20 (light) $\mathrm{K}$ of brightness temperature on a linear scale. The HI images have been convolved to $2^{\prime} \times 2^{\prime}$ angular resolution and the mean level of each image has been subtracted (using values shown in Fig. 9) for display purposes. The contours show 1.5, 3 , and $5 \mathrm{~K}$ of $1420 \mathrm{MHz}$ continuum brightness temperature. The continuum emission kernel of NRAO 655 is seen to coincide with a local minimum in $\mathrm{HI}$ emission; the correspondence is best in the middle of the velocity range shown 


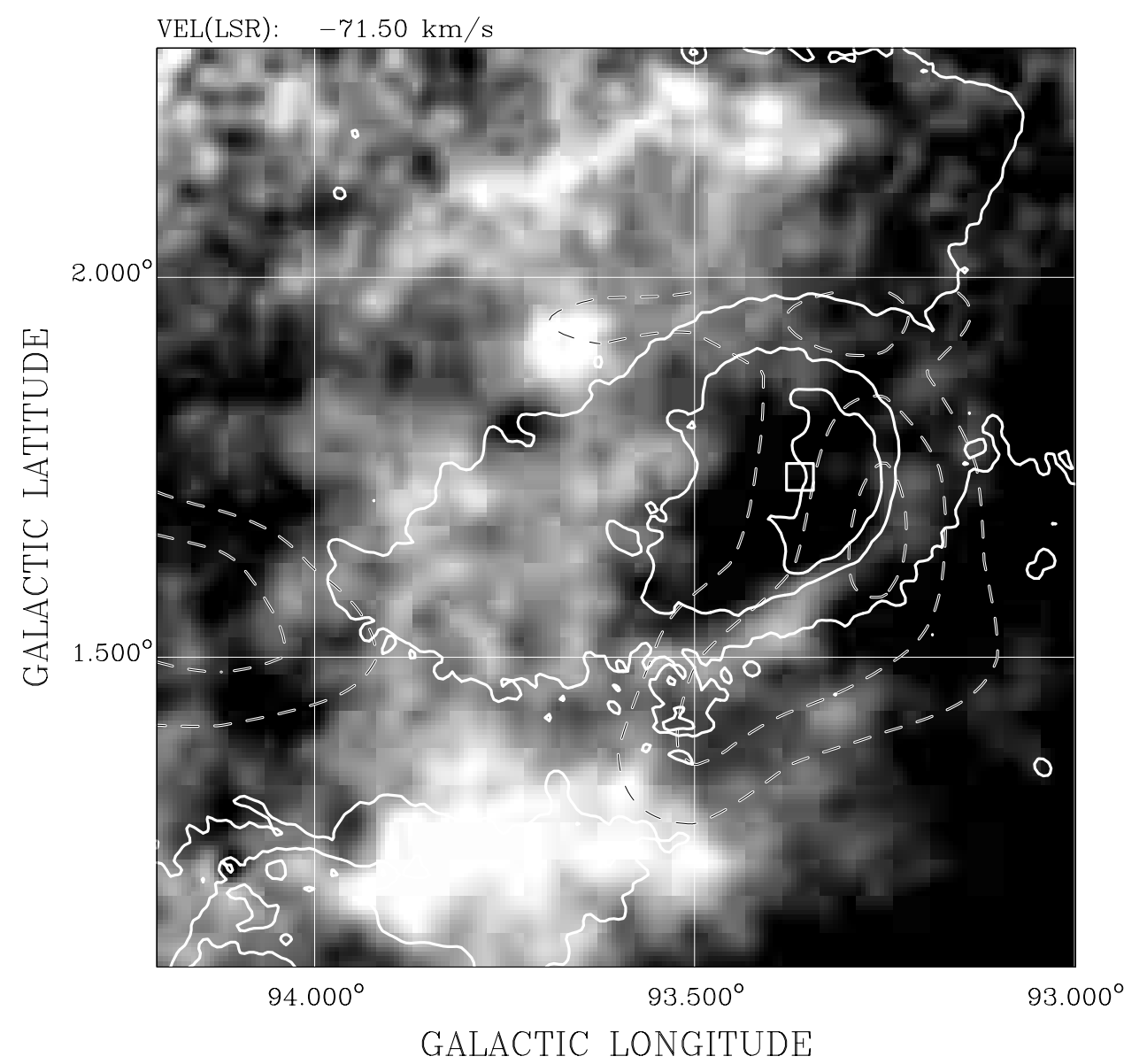

Fig. 8. $\lambda 21 \mathrm{~cm}$ HI emission (greyscale) and ${ }^{12} \mathrm{CO}(1-0)$ emission (dashed contours) centred on $-71.5 \mathrm{~km} \mathrm{~s}^{-1}$ and integrated over a $13 \mathrm{~km} \mathrm{~s}^{-1}$ velocity interval. The HI greyscale goes from 940 (dark) to 1270 (light) $\mathrm{K} \mathrm{km} \mathrm{s}^{-1}$ of $\int T_{b} \mathrm{~d} v$ on a linear scale. The CO contours are 13,26 , and $39 \mathrm{~K} \mathrm{~km} \mathrm{~s}^{-1}$ of $\int T_{b} \mathrm{~d} v$. The solid contours $\left(1.5,3\right.$, and $5 \mathrm{~K}$ of $T_{b}$ ) show $1420 \mathrm{MHz}$ continuum emission from NRAO 655. The CO contours appear to wrap around the head of NRAO 655, and the HI cavity coincides well with the kernel of NRAO 655. The small square denotes the centre used for the radial profiles shown in Fig. 12

west side of the head of NRAO 655, as shown in Fig. 8. Additional bright CO emission is seen east of NRAO 655, but this molecular gas is not thought to be important here because there is no evidence that NRAO 655 is interacting with it significantly.

Figure 7 is a montage of six grey-scale images of $\mathrm{HI}$ $21 \mathrm{~cm}$-line emission with overlaid contours of $1420 \mathrm{MHz}$ continuum emission from NRAO 655. The HI images have been interpolated onto a velocity grid of $2.6 \mathrm{~km} \mathrm{~s}^{-1}$ so that the six images span $13 \mathrm{~km} \mathrm{~s}^{-1}$. The mean level of each image has been subtracted so that the same grey-scale levels could be used for all images. A local minimum in HI emission is seen to coincide with the continuum-emission kernel of NRAO 655. The angular size of this minimum does not vary in a regular manner from channel to channel, as might have been expected if it were caused by an expanding shell of atomic gas. Instead, the contours of continuum emission complement the $21 \mathrm{~cm}$-line HI brightness quite well in the middle of the velocity range portrayed, and less well at the ends. The middle of this velocity range is $-71.5 \mathrm{~km} \mathrm{~s}^{-1}$. On this basis we can say that there is a cavity in the HI which matches the position and angular size of the kernel of NRAO 655, and that the velocity of this HI cavity is approximately $-71.5 \mathrm{~km} \mathrm{~s}^{-1}$.

Figure 8 shows both velocity-integrated $21 \mathrm{~cm}$-line $\mathrm{HI}$ emission (grey-scale) and velocity-integrated ${ }^{12} \mathrm{CO}(1-0)$ line emission (contours), with $1420 \mathrm{MHz}$ continuum contours as well. Both the $\mathrm{HI}$ emission and the $\mathrm{CO}$ emission have been integrated over a $13 \mathrm{~km} \mathrm{~s}^{-1}$ velocity interval centred on $-71.5 \mathrm{~km} \mathrm{~s}^{-1}$. The velocity-integrated CO contours appear to wrap around the head of NRAO 655 in the form of a crescent, and the cavity in the velocityintegrated HI emission coincides well in position and shape with the kernel of NRAO 655.

A $21 \mathrm{~cm}$-line HI emission profile is shown in Fig. 9. This profile was produced by using the mean brightness temperature at each velocity measured by the spectrometer in a circle of $1^{\circ}$ diameter centred at $(\ell, b)=(93.6,1.7)$ in the HI images. The large peak between -20 and $20 \mathrm{~km} \mathrm{~s}^{-1}$ is caused by local gas, the extended peak between -30 and $-80 \mathrm{kms}^{-1}$ is caused by the Perseus Arm, and the smallest peak near $-100 \mathrm{~km} \mathrm{~s}^{-1}$ is caused by the Outer Arm. The highest peak within the Perseus Arm is near $-67 \mathrm{~km} \mathrm{~s}^{-1}$. 




Fig. 9. $\lambda 21 \mathrm{~cm}$ atomic hydrogen emission profile representing the mean brightness temperature at 256 velocities, in a circle of $1^{\circ}$ diameter centred on $(\ell, b)=(93.6,1.7)$

Figure 10 shows contours of $\mathrm{H} \alpha+[\mathrm{NII}]$ optical emission overlaid on $1420 \mathrm{MHz}$ radio and $60 \mu \mathrm{m}$ infrared emission. The contours are $4 \sigma, 5 \sigma$, and $6 \sigma$ where $\sigma=$ $1.1510^{-18} \mathrm{~W} \mathrm{~m}^{-2}$ is the rms noise level in the optical image. This is the first detection of NRAO 655 optically. The integrated $\mathrm{H} \alpha+[\mathrm{NII}]$ emission within the $4 \sigma$ contour is $4.79 \pm 0.92 \mathrm{Jy}$.

\section{Discussion and interpretation}

\subsection{Flux density and spectral index}

To find the flux density of NRAO 655 at 1420 and $408 \mathrm{MHz}$ from the continuum images shown in Fig. 2, small-diameter sources were removed and the brightness of the diffuse emission was then integrated. The resulting flux densities of NRAO 655 are $11.8 \pm 0.5 \mathrm{Jy}$ and $11.6 \pm 1.0 \mathrm{Jy}$ at 1420 and $408 \mathrm{MHz}$, respectively. The uncertainties quoted include the uncertainty in establishing an accurate background level in each case. The flux density was also found at $2.695 \mathrm{GHz}$, by integrating the brightness of NRAO 655 in the map of Fürst et al. (1990); the value we obtained was $8.4 \pm 0.1 \mathrm{Jy}$. Combining these three values using linear regression gives a new value for the spectral index: $\alpha=-0.15 \pm 0.14$. This agrees within the errors with the value found by Goss et al. (1984) and confirms the classification of NRAO 655 as an optically thin thermal emitter.

\subsection{Kinematic distance}

Three radial or line-of-sight velocities for NRAO 655 are obtained from the results presented in Section III above. In Fig. 6, it is in the central three images that the ${ }^{12} \mathrm{CO}$ emission close to the continuum kernel of NRAO 655 is brightest, and we interpret this as showing that the molecular material reaches peak density near the kernel at $v \simeq$ $-72 \mathrm{~km} \mathrm{~s}^{-1}$. Figure 5 shows that the H158 $\alpha$ recombination line emission from HII in the head of NRAO 655, though spectrally broad, is centred on $-71 \mathrm{~km} \mathrm{~s}^{-1}$. Figure 7 shows that the continuum kernel of NRAO 655 matches an HI cavity in position and angular size, and that the velocity of this cavity is approximately $-71.5 \mathrm{~km} \mathrm{~s}^{-1}$. These three velocity determinations are in agreement; we therefore take the systemic velocity of the HII region, the HI cavity, and the adjacent molecular material as being $-71.5 \pm 1.5 \mathrm{~km} \mathrm{~s}^{-1}$.

Assuming purely circular motions in the Galactic Plane and a "flat" rotation curve with orbital velocity $v_{\mathrm{O}}$ for galactocentric radius $R$ exceeding that of the Sun, $R_{\mathrm{o}}$, the line-of-sight distance to an object having line-of-sight velocity $v_{\text {LofS }}$ at longitude $\ell$ is

$r_{\text {LofS }}=R_{\mathrm{o}}\left\{\cos \ell+\sin \ell\left[\left(\frac{v_{\mathrm{o}}}{v_{\mathrm{LofS}}+v_{\mathrm{o}} \sin \ell}\right)^{2}-1\right]^{\frac{1}{2}}\right\}$.

Using $v_{\text {LofS }}=-71.5 \mathrm{~km} \mathrm{~s}^{-1}$ and choosing $v_{\mathrm{O}}=220 \mathrm{~km} \mathrm{~s}^{-1}$ and $R_{\mathrm{O}}=8.5 \mathrm{kpc}$ gives the distance $r_{\text {LofS }}=8.8 \mathrm{kpc}$. The uncertainty in this distance is likely \pm 20 percent, given the uncertainties in the values of $v_{\text {LofS }}, v_{\mathrm{o}}$, and $R_{\mathrm{o}}$, and the possible existence of non-circular motions as rapid as $\simeq 10 \mathrm{~km} \mathrm{~s}^{-1}$. We adopt $8.8 \mathrm{kpc}$ as the kinematic distance of NRAO, whose linear dimensions are then $70 \times 130 \mathrm{pc}$. The corresponding galactocentric distance is $12.6 \mathrm{kpc}$.

\subsection{Ionization model based on free-free radio emission and $\mathrm{H} \alpha$ emission}

The radio continuum spectrum of NRAO 655 between 408 and $2695 \mathrm{MHz}$ is that of an optically thin thermal emitter. The theory of free-free radio emission (e.g. Rohlfs \& Wilson 1996) can therefore be used to construct an empirical model of the distribution of ionized gas. For an optically thin HII region, the brightness temperature (K) is approximately

$T_{b} \simeq 0.08235 T_{\mathrm{e}}^{-0.35} \nu^{-2.1} \int n_{\mathrm{e}}^{2} \mathrm{~d} l$

in which $\int n_{\mathrm{e}}^{2} \mathrm{~d} l$ is the emission measure $E M\left(\mathrm{~cm}^{-6} \mathrm{pc}\right)$, the integral is along the line of sight $(\mathrm{pc}), \nu$ is frequency $(\mathrm{GHz})$, and $T_{\mathrm{e}}$ is electron temperature $(\mathrm{K})$.

Though not unique, such a model is useful in establishing approximate electron densities $n_{\mathrm{e}}$ and masses $M$ of emitting material, and gives values for $E M$ and excitation parameter $U$. The optical depth at $1420 \mathrm{MHz}$ is at most $\tau_{1420} \simeq 0.001$ and at $408 \mathrm{MHz}$ it is at most $\tau_{408} \simeq 0.03$. 

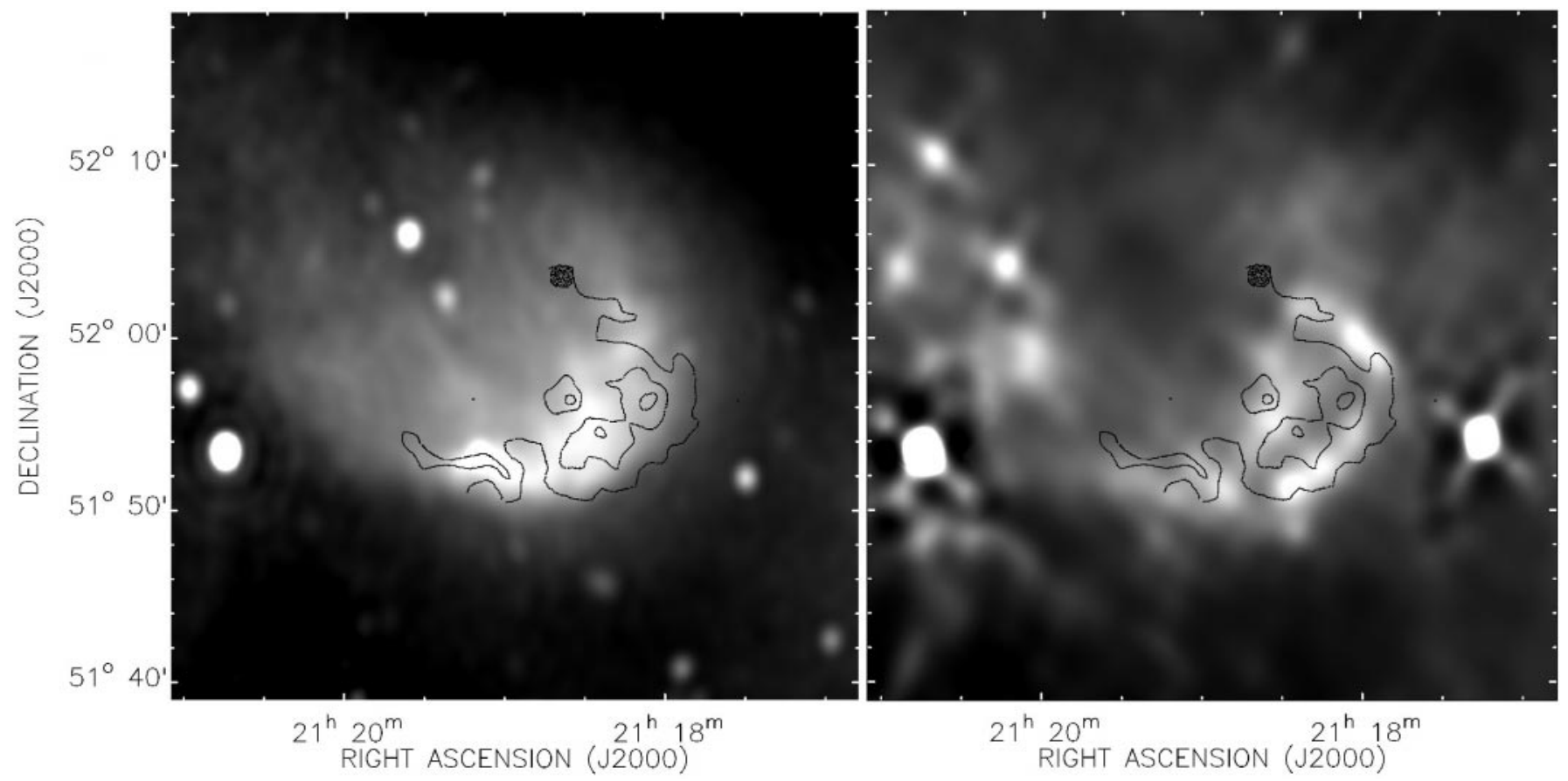

Fig. 10. $\mathrm{H}_{\alpha}+[\mathrm{NII}]$ optical emission contours overlaid on $1420 \mathrm{MHz}$ emission (left) and $60 \mu \mathrm{m}$ infrared emission (right) from the head of NRAO 655. The radio emission greyscale runs linearly from 1 (dark) to $6 \mathrm{~K}$ (light) of brightness temperature. The infrared emission greyscale runs linearly from 30 (dark) to $115 \mathrm{MJy} \mathrm{sr}^{-1}$ (light) of brightness

Table 3. Parameters of the four-component HII model

\begin{tabular}{|c|c|c|c|c|c|}
\hline Component & 1: Hemisphere & 2: Cylinder & 3: Ellipsoid & 4: Halo & Total \\
\hline Type & $\begin{array}{l}\text { thick-walled } \\
\text { hemisphere }\end{array}$ & $\begin{array}{c}\text { thick-walled } \\
\text { cylinder }\end{array}$ & $\begin{array}{c}\text { filled } \\
\text { ellipsoid }\end{array}$ & $\begin{array}{c}\text { thick-walled } \\
\text { ellipsoid }\end{array}$ & \\
\hline$n_{\mathrm{e}}\left(\mathrm{cm}^{-3}\right)$ & 8.5 & 8.5 & 5 & 2.6 & \\
\hline$S(\mathrm{Jy}) 1420 \mathrm{MHz}$ & 4.7 & 3.1 & 1.7 & 2.2 & $11.7 \mathrm{Jy}$ \\
\hline$S(\mathrm{Jy}) 408 \mathrm{MHz}$ & 5.2 & 3.5 & 2.0 & 2.5 & $13.2 \mathrm{Jy}$ \\
\hline$E M\left(\mathrm{~cm}^{-6} \mathrm{pc}\right)$ & 3285 & 2315 & 845 & 360 & \\
\hline$U\left(\mathrm{~cm}^{-2} \mathrm{pc}\right)$ & 90.0 & 78.5 & 64.6 & 70.4 & 122.3 \\
\hline $\operatorname{Mass}\left(M_{\odot}\right)$ & 13000 & 10900 & 7700 & 19000 & 50600 \\
\hline
\end{tabular}

Thus the approach is justified. Figure 11a shows a fourcomponent physical model (Foster 2000) constructed using software by Higgs et al. (1997) from a hemisphere, a cylinder, an ellipsoid, and a larger low-density ellipsoidal halo (Table 3 ). The gas was assumed to have uniform $T_{\mathrm{e}}$ of $7000 \mathrm{~K}$ (Churchwell \& Walmsley 1975). As Fig. 11a shows, the components are positioned to optimize the match to the observed 1420 and $408 \mathrm{MHz} T_{b}$ distributions shown in Figs. $2 \mathrm{a}$ and $2 \mathrm{~b}$. The model $T_{b}$ contours are overlaid on the actual 1420 and $408 \mathrm{MHz} T_{b}$ distributions in Figs. 11b and 11c.

As shown in Table 3, the total flux densities predicted by the model at 1420 and $408 \mathrm{MHz}$ are 11.7 and $13.2 \mathrm{Jy}$, respectively. The match with the observed values of $11.8 \pm$ 0.5 and $11.6 \pm 1.0 \mathrm{Jy}$, respectively, is good at $1420 \mathrm{MHz}$ and reasonably close at $408 \mathrm{MHz}$. The total mass of ionized gas is $510^{4} M_{\odot}$ at the assumed distance of $8.8 \mathrm{kpc}$. This mass includes 7 percent helium abundance by number.

It is clear from Fig. 8 that the kernel of the HII region coincides in position and shape with a cavity, or deficiency of HI. The "missing mass" of the atomic gas in the cavity can be estimated approximately. Taking the mean depth of the cavity to be $95 \mathrm{~K} \mathrm{~km} \mathrm{~s}^{-1}$, we can use the relation

$N_{\mathrm{HI}}=1.82310^{18} \int T_{b_{\mathrm{HI}}} \mathrm{d} v$

(e.g. Rohlfs \& Wilson 1996) and the solid angle of the cavity $(120 \mu \mathrm{sr})$ with the kinematic distance $(8.8 \mathrm{kpc})$ to find that the missing mass $\simeq 1.710^{3} M_{\odot}$ including 7 percent helium abundance. This value agrees within 30 percent with the estimate of the mass of material in the hemispherical component of NRAO 655 given in Table 3. This approximate agreement is satisfactory, given the difficulty of defining the precise boundary of the HI cavity and its depth; we estimate the uncertainty in the "missing" mass of the cavity to be $\pm 50 \%$.

Figure 10a shows contours of $\mathrm{H} \alpha+[\mathrm{NII}]$ emission drawn at the $4 \sigma, 5 \sigma$, and $6 \sigma$ levels superimposed on a greyscale representation of the $1420 \mathrm{MHz}$ continuum radio emission in the optical field (see Fig. 2a above). The $\mathrm{H} \alpha+[\mathrm{NII}]$ 




a

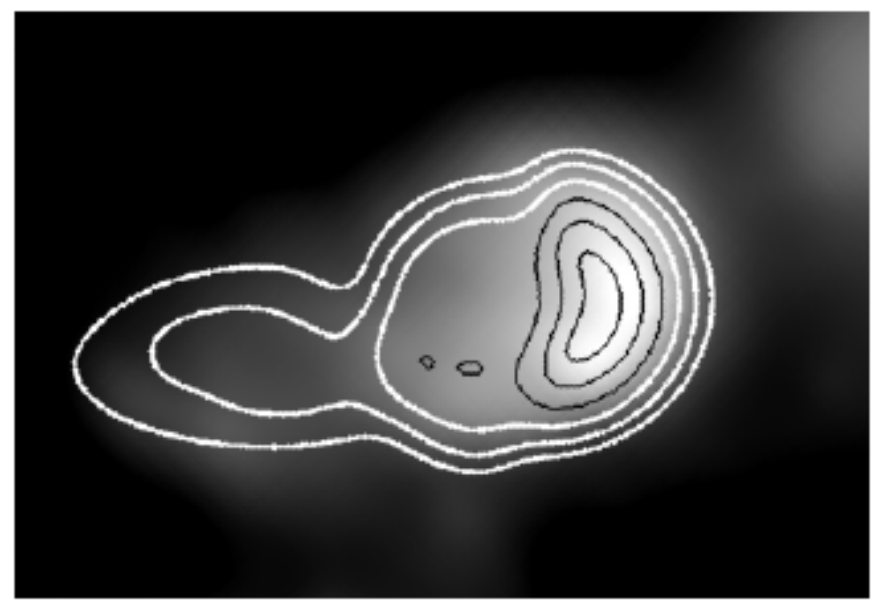

b

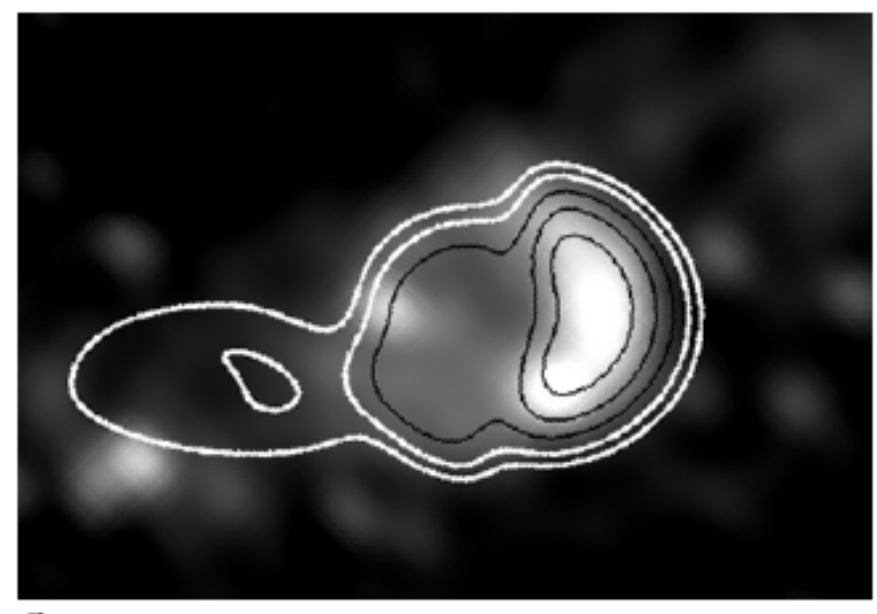

$\mathrm{c}$

Fig. 11. The four-component HII model (Table 3): a) $1420 \mathrm{MHz}$ emission from the model at very high resolution; b) $1420 \mathrm{MHz}$ emission from NRAO 655 with model contours overlaid; c) $408 \mathrm{MHz}$ emission from NRAO 655 with model contours overlaid

contours strongly resemble the morphology of the brightest radio emission from the kernel of NRAO 655, i.e. the portion of the kernel which lies closest to the molecular


Fig. 12. Radial profiles in $1420 \mathrm{MHz}$ continuum emission, $\lambda 21 \mathrm{~cm} \mathrm{HI}$ emission, and ${ }^{12} \mathrm{CO}(1-0)$ emission formed by azimuthally averaging over $90^{\circ}$ arcs in a quadrant centred within the kernel of NRAO 655. The centre of the arcs is indicated by a square in Fig. 8 . The quadrant extends to lower $\ell$ and lower $b$ from this symbol, i.e. it has mean position angle $225^{\circ}$ counterclockwise from the $b$-axis. The HI and CO emission are averaged over a $13 \mathrm{~km} \mathrm{~s}^{-1}$ interval centred on $-71.5 \mathrm{~km} \mathrm{~s}^{-1}$. The distributions of $1420 \mathrm{MHz}, \lambda 21 \mathrm{~cm} \mathrm{HI}$, and CO emission are seen to reach their peaks in sequence as radial distance from the square symbol increases

cloud shown in Fig. 8. (Contours from areas other than NRAO 655 have not been shown.) Similarly, Fig. 10b shows the same $\mathrm{H} \alpha+[\mathrm{NII}]$ contours superimposed on a greyscale representation of the $60 \mu \mathrm{m}$ HIRES infrared emission from the optical field (see Fig. 4 above). In this image we see clear anticorrelation between the $\mathrm{H} \alpha+[\mathrm{NII}]$ emission and the brightest infrared emission, i.e. those areas of NRAO 655 appear brighter optically which have less attenuation by the dust traced by the infrared emission.

The optical extinction along the $8.8 \mathrm{kpc}$ path between NRAO 655 and the Sun can be estimated. First we find the number of $\mathrm{HI}$ atoms and the number of $\mathrm{H}_{2}$ molecules per $\mathrm{cm}^{2}$ along the line of sight. Calculating the integrated brightness temperature under the HI velocity profile in Fig. 9 from the systemic velocity of NRAO 655, 
$-72 \mathrm{~km} \mathrm{~s}^{-1}$, to $+30 \mathrm{~km} \mathrm{~s}^{-1}$ (this includes all local HI on this line of sight) we find $N_{\mathrm{HI}}$ from Eq. (3) to be $1.0110^{22} \mathrm{~cm}^{-2}$. Similarly, the integrated brightness temperature under a ${ }^{12} \mathrm{CO}$ velocity profile gives the column density of $\mathrm{H}_{2}$ (Strong \& Mattox 1996):

$N_{\mathrm{H}_{2}}=1.910^{20} \int T_{b_{\mathrm{CO}}} \mathrm{d} v$

Integrating the $T_{b_{\mathrm{CO}}}(v)$ profile at the centre of the kernel of NRAO 655 over the same velocity range gives $N_{\mathrm{H}_{2}} \simeq 0.3810^{22} \mathrm{~cm}^{-2}$. Then the visual extinction $A_{\mathrm{v}} \simeq$ $5.310^{-22}\left(N_{\mathrm{H}_{\mathrm{I}}}+2 N_{\mathrm{H}_{2}}\right)$ (Savage \& Mathis 1979), which gives $A_{\mathrm{v}} \simeq 9.4$ magnitudes or $E(B-V)=2.96$ (Sudzius et al. 1996). This value for $A_{\mathrm{v}}$ is $\sim 2$ magnitudes larger than a similar estimate made by Vallée \& Higgs (1985) in the direction $(\ell, b)=(90.2,1.7)$.

Using the calibration factor obtained from the observations of NGC 6720, we find that the average $\mathrm{H} \alpha+[\mathrm{NII}]$ brightness of the portion of NRAO 655 within the $4 \sigma$ contours shown in Fig. $10(9.43 \mu \mathrm{sr})$ is $0.508 \mathrm{MJy} \mathrm{sr}^{-1}$. From this value, using the method of Reynolds (1988) the emission measure $E M=1.4 \pm 0.310^{5} \mathrm{~cm}^{-6} \mathrm{pc}$. Taking the equivalent radius of the bright $\mathrm{H} \alpha$-emitting solid angle to be $6^{\prime}$ or $15.2 \mathrm{pc}$, and assuming a volume filling factor $f$ of unity, we then find the rms electron density $n_{\mathrm{e}}$ to be $68 \pm 7 \mathrm{~cm}^{-3}$. This value of $n_{\mathrm{e}}$ is to be compared with the value $n_{\mathrm{e}}=8.5 \mathrm{~cm}^{-3} \mathrm{rms}$ used in the modelling to fit the 408 and $1420 \mathrm{MHz}$ radio continuum data in the head region (see above). The portions of NRAO 655 detected in the brightest regions of $\mathrm{H} \alpha$ emission are the portions with highest $n_{\mathrm{e}}$, so that the apparent discrepancy between the radio continuum and optical values of $n_{\mathrm{e}}$ is understood as a selection effect. In addition, the uncertainty quoted in the optically derived values of $E M$ and $n_{\mathrm{e}}$ do not include the rather large uncertainty in the optical extinction $A_{\mathrm{v}}$, or the uncertainty in the filling factor.

Confirmation that optical observations are indeed possible to great distances at low latitudes in this direction, even completely through the Galactic disk, comes from the fact that there are six "Zone of Avoidance Galaxies" (ZOAGs) seen within 5 degrees of NRAO 655 (Seeberger $\&$ Saurer 1998). The nearest ZOAG lies $3.1^{\circ}$ lower in $\ell$ and has $b \simeq 1.3^{\circ}$. Another lies $4.4^{\circ}$ higher in $\ell$ and has $b \simeq-0.6^{\circ}$. Thus it is not surprising that optical emission from bright HII regions at large distances is also visible.

In Fig. 3, the dashed profile which was fitted by eye to the observed $T_{b}$ profile has the functional form $T_{b}=5 \mathrm{e}^{-0.018(r-12)}$, in which $r$ is in pc. Because the HII constituting the tail is optically thin at $1420 \mathrm{MHz}$, $T_{b} \simeq \tau T_{\mathrm{e}}$, where $\tau$ is the optical depth and $T_{\mathrm{e}}$ is the electron temperature of the ionized gas. But $T_{\mathrm{e}} \simeq$ constant along the length of the outflow, and assuming also that the line-of-sight thickness $\simeq$ constant along the outflow, $\tau \propto n_{\mathrm{e}}^{2}$ (e.g. Rohlfs \& Wilson 1996). We therefore have an indication of the variation of $n_{\mathrm{e}}$ along the outflow, i.e. $n_{\mathrm{e}} \propto T_{b}^{1 / 2} \propto \mathrm{e}^{-0.009(r-12)}$.

\subsection{Molecular cloud}

The density of the molecular cloud can be found by use of (4). Taking the position of maximum $\int T_{b_{\mathrm{CO}}} \mathrm{d} v$ in Fig. 8, i.e. the portion of the molecular cloud adjacent to the brightest radio continuum emission in the kernel, we find $\int T_{b_{\mathrm{CO}}} \mathrm{d} v \simeq 26 \mathrm{~K} \mathrm{~km} \mathrm{~s}^{-1}$ above the general background in the interval -78 to $-65 \mathrm{~km} \mathrm{~s}^{-1}$. Hence $N_{\mathrm{H}_{2}}=4.910^{21} \mathrm{~cm}^{-2}$ along the line of sight through the cloud. Approximating the thickness of the cloud to be equal to its width in the plane of the sky (using $F W H M \simeq 11^{\prime}$ after deconvolution of the $8.7^{\prime}$ telescope beam), we find the cloud thickness to be $28 \mathrm{pc}$. Then the average density within the cloud along this line of sight is $58 \mathrm{H}_{2}$ molecules $\mathrm{cm}^{-3}$. The average mass density in $\mathrm{H}_{2}$ is then $1.910^{-22} \mathrm{~g} \mathrm{~cm}^{-3}$. Finally, since the average density within the FWHM of the Gaussian distribution along the line of sight is $58 \mathrm{~cm}^{-3}$, the peak $\mathrm{H}_{2}$ density is $71 \mathrm{~cm}^{-3}$. To find the total mass of the molecular cloud, we can estimate the volume of the cloud, again using Fig. 8, by "straightening out" the crescent form of the cloud and approximating it as an ellipsoid of height $190 \mathrm{pc}$ and maximum diameter $28 \mathrm{pc}$, so that the volume is roughly $7.810^{4} \mathrm{pc}^{3}$. Using the average density found above, we find the mass of $\mathrm{H}_{2}$ in the ellipsoid to be $2.210^{5} M_{\odot}$, and correcting for 7 percent numerical helium abundance, the total mass $\simeq 2.810^{5} M_{\odot}$. This is clearly an underestimate of the true mass since no ${ }^{12} \mathrm{CO}$ data were available above $b=2^{\circ}$, and the molecular cloud does appear to extend above this limiting latitude. It may be a further underestimate since the cloud may not lie in the plane of the sky.

\subsection{NRAO 655's age and exciting stars}

The elongated head-tail structure of NRAO 655, and its proximity to a molecular cloud, are consistent with formation of this HII region with a champagne flow. We suggest that ionized material has rushed into the intercloud medium from the eastern edge of the molecular cloud which we identified in Sect. 3 .

(i) Champagne-flow expansion in the slow regime: in the expansion phase of an HII region located on the margin of a molecular cloud, if the ionization front (IF) encounters a density profile approximated as a power law $r^{-w}$, there is a well-defined critical exponent $w_{\text {crit }}=3 / 2$ above which the IF cannot be trapped, so that it will continue as a supersonic R-type front. The outflowing gas acquires the same temperature everywhere, the pressure gradient follows the density gradient, and the entire cloud is set into motion (Rodríguez-Gaspar et al. 1995). Simultaneously, a rarefaction wave (RW) travels back into the molecular cloud. The side of the Strömgren sphere opposite the champagne flow, assumed to be ionization bounded rather than density bounded, is unaffected by the champagne flow and continues to evolve by the standard Strömgren process. 
The head-tail morphology of NRAO 655 strongly suggests a champagne flow in the East and an ionization front working into the molecular gas in the West. The fact that the "tail" follows an exponential decrease in density (Fig. 3) over an extended distance $(\sim 100 \mathrm{pc})$ is a signature of champagne outflow (Tenorio-Tagle 1982); such an exponential decrease signifies the reverse passage of the RW, and completely obliterates any evidence of even an abrupt pre-shock cloud edge.

For $\frac{3}{2}<w \leq 3$ (the "slow regime") a weak shock of almost constant velocity is driven outwards through the increasingly tenuous cloud, whereas for $w>3$ the shock is strong and accelerates. (We argue below for a value of $w$ of $\simeq 3 / 2$.) Franco et al. (1990), assuming a power-law profile $n=n_{\max }\left(r / r_{\mathrm{c}}\right)^{-w}$, give an approximate relation for the position $r(t)$ of the shock and IF at the leading edge of the champagne flow when $\frac{3}{2} \leq w<3$ :

$r(t) \simeq r_{\mathrm{c}}+\left\{1+\left(\frac{3}{3-w}\right)^{1 / 2}\right\} \cdot c_{\mathrm{s}} \cdot t$.

Here $c_{\mathrm{s}}$ is the sound speed in the ionized gas and $r_{\mathrm{c}}$ is the radius of a constant-density core within the molecular cloud.

To estimate $w$ in the parent molecular cloud of NRAO 655, we argue simply that the lowest permissible value of $w$ should be chosen $(w \simeq 3 / 2)$. There are two grounds for this choice. First, the NRAO 655 outflow is very long $(\sim 100 \mathrm{pc})$, and the peak CO intensity in Fig. 8 occurs adjacent to the kernel of NRAO 655 , and corresponds to a maximum of only $n_{\mathrm{H}_{2}} \simeq 71 \mathrm{~cm}^{-3}$ (see above). Thus the power law must be "weak" to be valid over its entire extent; if $w$ is set too high, the density pre-existing at the east end of the outflow becomes unrealistically small. Second, we assert that a champagne outflow has indeed occurred; hence $w$ cannot be lower than $3 / 2$.

With $w \simeq 3 / 2$, (5) shows the speed of the outflow to be $(1+\sqrt{2}) c_{\mathrm{s}} \simeq 26 \mathrm{~km} \mathrm{~s}^{-1} \simeq 26.510^{-6} \mathrm{pc} \mathrm{yr}^{-1}$. Therefore the $100 \mathrm{pc}$ outflow must have age $\simeq 3.8 \mathrm{Myr}$. We then see that the choice of $w \simeq 3 / 2$ was not sensitive, since a change in $w$ of 0.25 would be required to change the calculated age by only 5 percent.

(ii) Champagne-flow mass balance: the material in the tail of the outflow must be constantly re-ionized: even at a location in the tail where $n_{\mathrm{e}}$ is small (e.g. $2 \mathrm{~cm}^{-3}$ ), the recombination time-scale with $T_{\mathrm{e}} \simeq 7000 \mathrm{~K}$ is $\tau_{\mathrm{R}} \simeq$ $1200 T_{\mathrm{e}}^{1 / 2} n_{\mathrm{e}}^{-1} \simeq 510^{4} \mathrm{yr}$ (Schwartz 1973). Thus the star(s) in the kernel producing the champagne outflow must also continually re-ionize the tail with Lyman continuum photons. Then the Lyman continuum flux $F_{\star}$ from the $\operatorname{star}(\mathrm{s})$ in the kernel must sustain $U_{\text {total }}=$ $\left(U_{1}^{3}+U_{2}^{3}+U_{3}^{3}\right)^{1 / 3}=114 \mathrm{~cm}^{-2}$ pc. (We omit the large halo in Table 3 , on the grounds that the halo material is not involved in the champagne outflow.)

A single star capable of this would have spectral type O4 (Panagia 1973). However, its main-sequence lifetime
$t_{\mathrm{MS}}$ is only 3.0 Myr (Chiosi et al. 1978). This is smaller than the estimate of the age of the outflow above, which is a major inconsistency. We can calculate the age of the outflow in another way, however, to check its correctness.

Knowledge of the mass of ionized gas in NRAO 655 (Table 3) can be used to estimate its age. Franco et al. (1994) derive the rate of expansion of an ionization front working into the ionization-bounded side of a blister, by considering the balance of creation and expulsion of ionized material. The growth of the ionized region inside the cloud (the head) is controlled by the balance between increasing ionization density caused by the advance of the ionization front into the molecular gas, and decreasing ionization density caused by the outflow into the tail. Taking $n_{\mathrm{o}}$ as the number density of the molecular gas and $R_{\mathrm{o}}$ as the initial Strömgren radius, they find the total mass of ionized material within the kernel and the tail at age $t$ to be

$M_{\mathrm{i}}(t) \simeq \frac{2 \pi}{3} R_{\mathrm{o}}^{3} \mu_{\mathrm{p}} n_{\mathrm{o}}\left[\left(1+\frac{5 c_{\mathrm{s}} t}{2 R_{\mathrm{o}}}\right)^{\frac{6}{5}}-1\right]$

in which $\mu_{\mathrm{p}}$ is the mass of the proton. $R_{\mathrm{o}}$ depends on $F_{\star}$ and on the possible attenuation of this flux by dust within the kernel; we assume optical depth $\tau_{\mathrm{d}}$ for the dust. Then the expression for $R_{\mathrm{O}}$ becomes

$R_{\mathrm{o}}=\left(\frac{3 F_{\star} \mathrm{e}^{-\tau_{d}}}{4 \pi \alpha_{\mathrm{B}}\left(2 n_{\mathrm{o}}\right)^{2}}\right)^{1 / 3}$

where $\alpha_{\mathrm{B}}$, the recombination coefficient, has the value $3.5210^{-13} \mathrm{~cm}^{3} \mathrm{~s}^{-1}$ at $7000 \mathrm{~K}$ (Storey \& Hummer 1995) and we take the value of $F_{\star}$ from Panagia (1973).

Franco et al. (1994) derive an expression for the timedependence of the radius $R_{\mathrm{i}}$ of the ionization front eroding into the molecular gas:

$R_{\mathrm{i}}(t) \simeq R_{\mathrm{o}}\left(1+\frac{5}{2} \frac{c_{\mathrm{s}} t}{R_{\mathrm{o}}}\right)^{2 / 5}$

Eliminating $t$ between (6) and (8) we find an expression for the original molecular density in terms of $M_{\mathrm{i}}$ :

$n_{\mathrm{o}}=\frac{3 M_{\mathrm{i}}}{4 \pi \mu_{\mathrm{p}} R_{\mathrm{i}}^{3}}\left\{1+\left[1+\frac{\pi F_{\star} \mathrm{e}^{-\tau_{\mathrm{d}}} \mu_{\mathrm{p}}^{2} R_{\mathrm{i}}^{3}}{3 \alpha_{\mathrm{B}} M_{\mathrm{i}}^{2}}\right]^{\frac{1}{2}}\right\}$.

To find the age of the outflow, one assumes a stellar spectral type, hence $F_{\star}$, and a value for $\tau_{\mathrm{d}}$. Using the known mass $M_{\mathrm{i}}$ and the observed radius $R_{\mathrm{i}}$, one finds $n_{\mathrm{o}}$ from (9) and hence $R_{\mathrm{o}}$ from (7). Then, rearranging (8), the age of the outflow is found from

$t=\frac{2 R_{\mathrm{o}}}{5 c_{\mathrm{s}}}\left[\left(\frac{R_{\mathrm{i}}}{R_{\mathrm{o}}}\right)^{5 / 2}-1\right]$

The question is then whether this age is compatible with $t_{\mathrm{MS}}$, the main-sequence lifetime of the stellar type assumed.

In our case we use $\tau_{\mathrm{d}}=0.92$ ( 1 magnitude), $M_{\mathrm{i}}=$ $31600 M_{\odot} / 1.28 \simeq 25000 M_{\odot}$ (the combined mass excluding helium, of components 1,2 , and 3 in Table 3 ), and the 
observed radius of the kernel, $R_{\mathrm{i}}=24 \mathrm{pc}$. (We are assuming that by far the largest part of the ionized mass in the head resides in the kernel, i.e. the mass of the kernel $\simeq$ $13000 M_{\odot}$, which is the mass of component 1 in Table 3 .) For a star of spectral type O4, we find $n_{\mathrm{o}}=35 \mathrm{~cm}^{-3}$. The value for $n_{\mathrm{o}}$ can be compared with our CO observations: the value of $n_{\mathrm{o}}$ at the approximate location of the exciting star (e.g. the small square in Fig. 8) can be found by approximating the observed distribution of $\int T_{\mathrm{B}_{\mathrm{CO}}} \mathrm{d} v$ in Fig. 8 as Gaussian with $F W H M \simeq 14^{\prime}$, and estimating that the square lies $8^{\prime}$ from the peak of the Gaussian. Since $n_{\mathrm{o}}$ at the peak is $71 \mathrm{~cm}^{-3}$ (see above), we find $n_{\mathrm{o}}$ at the location of the square to be $29 \mathrm{~cm}^{-3}$. The values of $n_{\mathrm{o}}$ are therefore in approximate agreement. However, for a star of spectral type $\mathrm{O} 4$, the age of the outflow is found to be $t=8 \mathrm{Myr}$. This age is again much longer than $t_{\mathrm{MS}}$ of an O4 star, or of any O star (Chiosi et al. 1978).

Alternatively, a number $N$ of stars of somewhat later spectral type may be present in the kernel of NRAO 655 . Together they must contribute the Lyman continuum flux required to account for $U_{\text {total }}=114 \mathrm{~cm}^{-2} \mathrm{pc}$, but the value of $F_{\star}$ used in $(7)$ must be appropriate to the later spectral type, and $R_{\mathrm{O}}$ is therefore reduced. Most importantly, each of the $N$ stars produces its own Strömgren sphere which expands into the surrounding medium, encounters the density gradient, and produces its own champagne outflow, where the mass involved in each outflow is $N$ times smaller than the previous value of $M_{\mathrm{i}}$. Thus in (9) we now use $M_{\mathrm{i}} / N$ and Eq. (10) gives the common age of each of the $N$ simultaneous outflows. The ejected mass in each of the $N$ outflows combines to form the observed tail. We again use $\tau_{\mathrm{d}}=0.92$ and total mass of ionized hydrogen $M_{\mathrm{i}} \simeq 25000 M_{\odot}$. The observed radius of the kernel is $24 \mathrm{pc}$; however, if the $N$ stars are randomly distributed throughout the volume of a sphere, the appropriate value to use in (9) is $R_{\mathrm{i}} / N^{1 / 3}$. For example, with stars of spectral type 08.5 , we require 22 stars to provide $U_{\text {total }}=114 \mathrm{~cm}^{-2} \mathrm{pc}$, each ionizes $1140 M_{\odot}$ of hydrogen, we use $R_{\mathrm{i}}=8.6 \mathrm{pc}$ in (9), and we find $n_{\mathrm{o}}=35 \mathrm{~cm}^{-3}$. Again, $n_{\mathrm{o}}$ agrees with our observations, approximately. (Eq. (9) is only weakly dependent on $F_{\star}$.) Then from (10) the age of each outflow is $3.4 \mathrm{Myr}$. This age is compatible with $t_{\mathrm{MS}}$ of an 08.5 star, which is $\simeq 3.6 \mathrm{Myr}$. This calculation can be repeated for a range of spectral types, but it is found that $\mathrm{O} 8.5$ is the earliest spectral type for which the age of the outflow is indeed less than $t_{\mathrm{MS}}$.

The main conclusion from the foregoing is that a single very early type star is not compatible with the mass and size and therefore the age of the NRAO 655 outflow, and instead a cluster containing several longer-lived stars (O8.5 or later) is required. The second conclusion is that the age of the outflow is found by this mass-balance argument to be $\geq 3.4 \mathrm{Myr}$, which is compatible with the previous estimate of 3.8 Myr from analysis of the champagneflow in the slow regime.

It is important to check the kinetic energy of the outflowing material against the energy available in the Lyman continuum flux from the stars over the lifetime of the outflow. If the entire mass of components 1, 2, and 3 listed in Table $3\left(3.1610^{4} M_{\odot}\right)$ is moving outward at $26 \mathrm{~km} \mathrm{~s}^{-1}$ as suggested above, its kinetic energy is $2.110^{50} \mathrm{erg}$. In comparison, an O8.5 star has Lyman continuum luminosity of $21500 L_{\odot}$ (Panagia 1973), and over the suggested 3.8 Myr duration of the outflow 22 of these would have been capable of imparting $\sim 2.210^{53}$ erg to the gas if every photon were absorbed by the gas. Thus the efficiency of conversion from ultraviolet flux to kinetic energy of the outflow need be no higher than 0.1 percent. Very large amounts of stellar energy remain for ionization, dissociation, heating, and radiative losses.

(iii) Molecular dissociation in the West: whereas the radially decreasing gas density on the east side of NRAO 655's kernel is responsible for the champagne flow discussed above, the gas density on the west side of the kernel increases with radial distance from the kernel. No champagne flow can form, and the HII region develops very asymmetrically, as observed. However, an ionization front works into the adjacent molecular cloud. Outside the ionization front, the flux of ultraviolet photons from the exciting stars, though low-pass filtered by passage through the ionization front, is still capable of dissociating the $\mathrm{H}_{2}$ to form HI. As a result, a layer of atomic gas should form between the ionization front and the molecular cloud.

Roger \& Dewdney (1992) have analyzed this situation. The dissociation front which forms between the HI zone and the $\mathrm{H}_{2}$ zone will in general advance through the interstellar medium until equilibrium is reached between the rate of the dissociation process, powered by the flux of Lyman-Werner photons from an exciting star, and the rate at which $\mathrm{H}_{2}$ can re-form on dust grains. Before equilibrium of the dissociation front is reached, however, it may be overtaken by the star's ionization front and associated shock ("catch-up"). Stars in the range B2.5 to O5 are studied. Amongst the findings are that (i) the HI zone formed by dissociation persists unshocked for millions of years, and (ii) larger masses of HI are produced before catch-up occurs in environments of low molecular density rather than high.

In the case of NRAO 655, we do see a partial shell of HI located between the HII of the kernel and the $\mathrm{H}_{2}$ of the molecular cloud (see Fig. 8). Figure 12 shows radial profiles of emission from HII, HI, and molecular gas versus radial distance from the location of the square drawn in Fig. 8. The three gas components peak sequentially as distance from the centre increases, as expected if the HI is formed by dissociation of the $\mathrm{H}_{2}$ in the molecular cloud by stars in the kernel. After the HII emission peaks, the HI emission peaks, showing the location of the partial HI shell. Here the flux of ionizing photons $(\lambda<91.2 \mathrm{~nm})$ from the exciting stars diminishes, but lower-energy photons are still present to dissociate the $\mathrm{H}_{2}$. The flux of low-energy photons becomes insufficient for appreciable dissociation at larger radii. 
In the previous section we argued that instead of a single star of very early type ionizing the the kernel and producing the eastward outflow, it is more likely that there are several stars of later spectral type located in the volume of the kernel. This suggestion is compatible with the requirements for the stars needed to produce the lowerenergy uv flux for dissociation of $\mathrm{H}_{2}$ into the partial $\mathrm{HI}$ shell which we observe, since stars of later spectral type have longer $t_{\mathrm{MS}}$, and also have lower ionizing flux, which results in later catch-up. Thus the stellar requirements for molecular dissociation in the West are compatible with those for production of the ionized champagne outflow in the East.

We can estimate the mass of atomic gas in the partial HI shell seen in Fig. 8. Estimating that the average value of $\int T_{b} \mathrm{~d} v$ at $\lambda 21 \mathrm{~cm}$ of the shell above the local background $\simeq 125 \mathrm{~K} \mathrm{~km} \mathrm{~s}^{-1}$, Eq. (3) gives $N_{\mathrm{HI}}=2.2810^{20} \mathrm{~cm}^{-2}$. Taking the thickness of the shell $\simeq 8 \mathrm{pc}$ and the radius $\simeq 30 \mathrm{pc}$, the line-of-sight path length through the shell $\simeq 20 \mathrm{pc}$. Then the average HI density in the shell $\simeq 3.7 \mathrm{~cm}^{-3}$. Assuming that $\sim \frac{1}{4}$ of a complete spherical shell is present, we then find the mass of $\mathrm{HI}$ in the shell $\simeq 210^{3} M_{\odot}$. Correcting for 7 percent helium atoms by number gives a total atomic mass of $\simeq 2.610^{3} M_{\odot}$.

\section{Conclusions}

NRAO 655 has been conclusively shown to be a thermal object, and three observational approaches have converged to yield a kinematic distance. First, the HI-line images reveal a cavity in the interstellar $\mathrm{HI}$ at $-71.5 \mathrm{~km} \mathrm{~s}^{-1}$ which matches the HII kernel of NRAO 655 well in size and position (see Fig. 8). Second, molecular gas mapped in ${ }^{12} \mathrm{CO}(1-0)$ emission shows a molecular cloud whose brightest emission occurs adjacent to the kernel of NRAO 655, with central velocity $-72 \mathrm{~km} \mathrm{~s}^{-1}$ (see Fig. 6). Third, we detect the HII region in $\mathrm{H} 158 \alpha$ recombination line emission, and find a central velocity of $-71 \mathrm{~km} \mathrm{~s}^{-1}$ (see Fig. 5). These three velocities agree, and the kinematic distance to the $\mathrm{HI}-\mathrm{HII}-\mathrm{H}_{2}$ complex is therefore found to be $8.8 \mathrm{kpc}$. The linear extent of the radio-emitting object $\simeq 70 \times 130$ pc. We also detect NRAO 655 optically for the first time, in $\mathrm{H} \alpha+[\mathrm{NII}]$ emission.

We have suggested that the eastward extension of NRAO 655 originates in a champagne outflow, and two approaches were taken in estimating its age. In the first, it was assumed that the initial density of the molecular cloud decreased as a weak power law $\sim r^{-w}$, so that the velocity of the ionization front $\simeq(1+\sqrt{2}) c_{\mathrm{s}} \simeq 26 \mathrm{~km} \mathrm{~s}^{-1}$, and thus the outflow age $t \simeq 3.8 \mathrm{Myr}$. In the second, the ionized mass-balance approach was used to show that a single early-type star cannot be responsible for an outflow of the size and mass of NRAO 655, whereas a group of later-type stars would suffice since their $t_{\mathrm{MS}}$ can exceed the age of the outflow. We therefore estimate the age of the outflow $\simeq 3.8 \mathrm{Myr}$, the mass $\simeq 310^{4} M_{\odot}$, the length $\simeq 100 \mathrm{pc}$, and the group of central stars as type $\mathrm{O} 8.5$ or later.
We further suggested that the partial HI shell observed adjacent to the kernel of NRAO 655 has been formed by dissociation of $\mathrm{H}_{2}$ in the molecular cloud, and found that the stellar requirements for the dissociation process (i.e. later-type stars rather than earlier) were generally compatible with those required for the champagne outflow to the East. The mass of atomic gas in the partial shell $\simeq 2.610^{3} M_{\odot}$.

Acknowledgements. It is a pleasure to thank J. A. Galt for his unstinting efforts on our behalf in preparing the DRAO 26-metre telescope for our recombination line observations. We also thank K. Tapping for his help in the same observations. We are very grateful to Tom Dame for providing the ${ }^{12} \mathrm{CO}$ data from the Harvard-Smithsonian CO survey in advance of publication. The DRAO export software package has been very useful in this work, and we thank L. A. Higgs and A. G. Willis for their tireless efforts in making it available to the community. The Canadian Galactic Plane Survey (CGPS) is a Canadian project with international partners. It is described by Taylor et al. (2000) with additional information available online at www.ras.ucalgary.ca/CGPS, and is supported by a grant from the Natural Sciences and Engineering Research Council of Canada (NSERC). The Dominion Radio Astrophysical Observatory is operated as a national facility by the National Research Council of Canada. We thank Brad Wallace for his help in providing us with the CGPS data for the J8 field in pre-release form. This work was supported by an NSERC research grant to DR.

\section{References}

Arendt, R. G. 1989, ApJS, 70, 181

Baars, J. W. M., Genzel, R., Pauliny-Toth, I. I. K., \& Witzel, A. 1977, A\&A, 61, 99

Cao, Y., Terebey, S., Prince, T. A., \& Beichman, C. A. 1997, ApJS, 111, 387

Chiosi, C., Nasi, E., \& Sreenivasan, S. R. 1978, A\&A, 63, 103

Churchwell, E., \& Walmsley, C. M. 1975, A\&A, 38, 451

Clark, B. G. 1980, A\&A, 89, 377

Dame, T. M., Hartmann, Dap, \& Thaddeus, P. 2000, ApJ, submitted

Fich, M. 1983, Ph.D. Thesis, University of California, Berkeley Fich, M. 1986, AJ, 92, 787

Foster, T. J. 2000, M.Sc. Thesis, Department of Physics, University of Alberta

Franco, J., Shore, S. N., \& Tenorio-Tagle, G. 1994, ApJ, 436, 795

Franco, J., Tenorio-Tagle, G., \& Bodenheimer, P. 1990, ApJ, 349,126

Fürst, E., Reich, W., Reich, P., \& Reif, K. 1990, A\&AS, 85, 691

Galt, J. A., \& Kennedy, J. E. D. 1968, AJ, 73, 135

Goss, W., Mantovani, F., Salter, C. J., Tomasi, P., \& Velusamy, T. 1984, A\&A, 138, 469

Green, D. A. 1984, MNRAS, 209, 449

Green, D. A. 1991, PASP, 103, 209

Haslam, C. G. T., Salter, C. J., Stoffel, H., \& Wilson, W. E. 1982, A\&AS, 47, 1

Hawley, S. A., \& Miller, J. S. 1977, ApJ, 212, 94

Higgs, L. A. 1999, in New Perspectives on the Interstellar Medium, ASP Conf. Ser., vol. 168, Astronomical Society of the Pacific, San Francisco, ed. A. R. Taylor, T. L. Landecker, \& G. Joncas, 15 
Higgs, L. A., Hoffmann, A. P., \& Willis, A. G. 1997, Astronomical Data Analysis Software and Systems VI, ASP Conf. Ser., vol. 125, ed. G. Hunt, \& H. E. Payne, 58

Higgs, L. A., \& Tapping, K. F. 2000, AJ, 120, 2471

Higgs, L. A., Vallée, J. P., Albinson, J. S., Batrla, W., \& Goss, W. M. 1987, A\&A, 181, 351

Kallas, E., \& Reich, W. 1980, A\&AS, 42, 227

Landecker, T. L., Dewdney, P. E., Burgess, T. A., et al. 2000, A\&AS, 145, 509

Landecker, T. L., Higgs, L. A., \& Roger, R. S. 1985, AJ, 90, 1082

Mantovani, F., Nanni, M., Salter, C. \& Tomasi, P. 1982, A\&A, 105,176

Miralles, M. P., Rodriguez, L. F., \& Scalesi, E. 1994, ApJ, 92,173

Molinari, S., Brand, J., Cesaroni, R., \& Palla, F. 1996, A\&A, 308,573

Panagia, N. 1973, AJ, 78, 929.

Pauliny-Toth, I. I. K., Wade, C. M., \& Heeschen, D. S. 1966, A\&AS, 13, 65

Reich, W., Reich, P., Fürst, E., \& Reif, K. 1990a, A\&AS, 85,633

Reich, W., Reich, P., \& Fürst, E. 1990b, A\&AS, 83, 539

Reynolds, R. J. 1988, ApJ, 333, 341

Rodríguez-Gaspar, J. A., Tenorio-Tagle, G., \& Franco, J. 1995, ApJ, 451, 210

Roger, R. S., \& Dewdney, P. E. 1992, ApJ, 385, 536.
Rohlfs, K., \& Wilson, T. L. 1996, Tools of Radio Astronomy, $2^{\text {nd }}$ edition (Springer-Verlag, Berlin)

Savage, B. D., \& Mathis, J. S. 1979, ARA\&A, 17, 73

Schwartz, J. 1973, ApJ, 182, 449

Seeberger, R., \& Saurer, W. 1998, A\&AS, 127, 101

Steer, D. G., Dewdney, P. E., \& Ito, M. R. 1984, A\&A, 137, 159

Stetson, P. B. 1987, PASP, 99, 191

Storey, P. J., \& Hummer, D. G. 1995, MNRAS, 272, 41

Strong, A. W., \& Mattox, J. R. 1996, A\&A, 308, L21

Sudzius, J., Bobinas, V., \& Raudeliunas, S. 1996, Baltic Astron., 5, 485

Taylor, A. R. 1999, in New Perspectives on the Interstellar Medium, ASP Conf. Ser., vol. 168, Astronomical Society of the Pacific, San Francisco, ed. A. R. Taylor, T. L. Landecker, \& Joncas, 3

Taylor, A. R., Dewdney, P. E., Landecker, T. L., et al. 2000, $\mathrm{AJ}$, in preparation

Tenorio-Tagle, G., \& Bedijn, P. J. 1981, A\&A, 99, 305

Tenorio-Tagle, G. 1982, The dynamical evolution of HII regions in non-uniform environments, in Regions of Recent Star Formation, Astrophys. \& Space Sci. Library, vol. 93, ed. R. S. Roger, \& P. E. Dewdney (D. Reidel, Dordrecht, Holland), 1

Vallée, J. P., \& Higgs, L. A. 1985, AJ, 90, 2061

van der Werf, P., \& Higgs, L. A. 1990, A\&A, 235, 407

van der Werf, P., \& Higgs, L. A. 1991, A\&AS, 89, 599

Velusamy, T., \& Kundu, M. R. 1974, A\&A, 32, 375

Wouterloot, J. G. A., \& Brand, J. 1989, A\&AS, 80, 149 